\title{
Continuum models of cohesive stochastic swarms: the effect of motility on aggregation patterns
}

\author{
Barry D. Hughes ${ }^{\mathrm{a}, *}$, Klemens Fellner ${ }^{\mathrm{b}}$ \\ ${ }^{a}$ Department of Mathematics and Statistics, University of Melbourne, Victoria 3010, Australia \\ ${ }^{b}$ Institute for Mathematics and Scientific Computing, University of Graz, A-8010 Graz, Heinrichstr. 36, Austria
}

\begin{abstract}
Mathematical models of swarms of moving agents with non-local interactions have many applications and have been the subject of considerable recent interest. For modest numbers of agents, cellular automata or related algorithms can be used to study such systems, but in the present work, instead of considering discrete agents, we discuss a class of one-dimensional continuum models, in which the agents possess a density $\rho(x, t)$ at location $x$ at time $t$. The agents are subject to a stochastic motility mechanism and to a global cohesive inter-agent force. The motility mechanisms covered include classical diffusion, nonlinear diffusion (which may be used to model, in a phenomenological way, volume exclusion or other short-range local interactions), and a family of linear redistribution operators related to fractional diffusion equations. A variety of exact analytic results are discussed, including equilibrium solutions and criteria for unimodality of equilibrium distributions, full time-dependent solutions, and transitions between asymptotic collapse and asymptotic escape. We address the behaviour of the system for diffusive motility in the low-diffusivity limit for both smooth and singular interaction potentials and show how this elucidates puzzling behaviour in fully deterministic non-local particle interaction models. We conclude with speculative remarks about extensions and applications of the models.
\end{abstract}

Keywords:

swarms, cohesion, stationary states, long-range interactions, non-local aggregation equation, integro-partial differential equations, singular interaction potential

2000 MSC: 35K55, 45K05, 60K35, 82C70

\section{Introduction}

Mathematical models of swarms of moving agents with nonlocal interactions have many applications and have been the subject of considerable recent interest [1, 2, 3, 4]. For modest numbers of agents, cellular automata or related algorithms can be used to study such systems, but in the present work, instead of considering discrete agents, we shall examine a onedimensional continuum model, in which the agents possess a density $\rho(x, t)$ at location $x$ at time $t$.

In the evolution equation

$$
\frac{\partial \rho}{\partial t}+\frac{\partial}{\partial x}\left[\rho(x, t) \int_{-\infty}^{\infty} f(x-\xi) \rho(\xi, t) d \xi\right]=0
$$

if $f=-W^{\prime}$ is an odd function (associated with a symmetric potential $W$ ), one may interpret $f$ as an inter-agent force (scaled against a local resistance to motion) that induces a drift velocity for each agent relative to the instantaneous location of the rest of the swarm. The case of a double-well potential [5, 6, 7] has received comparatively little attention in the literature to date, and will be specially emphasised in the present paper. We shall only consider the case in which the inter-agent force is cohesive

\footnotetext{
${ }^{*}$ Tel.: +61383445557

Email addresses: barrydh@unimelb.edu .au (Barry D. Hughes), klemens.fellner@uni-graz.at (Klemens Fellner)
}

at large distances, that is, $f(x)<0$ and so $W^{\prime}(x)>0$, for all sufficiently large positive $x$ ), and we shall normally insist that the system has finite agent mass, that is

$$
C=\int_{-\infty}^{\infty} \rho(x, t) d x
$$

is finite, although for certain asymptotic arguments we shall relax this constraint briefly. Since Eq. (1) has the form of a continuity equation, it conserves mass, so $C$ is independent of time. For related references concerning the non-local interaction equation (1) with purely aggregating or repulsive potentials (and possible diffusion) we refer to the following papers: $[8,9,10,11,12,13,14,15,16,17,18]$.

If the interactions are cohesive at all inter-agent distances, one would expect a finite-mass swarm to collapse and ultimately occupy a small high-concentration region, with $\rho$ converging in an appropriate sense to a delta function. In agent-based simulations, complete collapse can be prevented by imposing volume exclusion, while for continuum modelling, collapse can be opposed by making the inter-agent force repulsive at short distances (see Carrillo et al. [9] and references therein, and Holm and Putkarazade [19, 20]). The consequences of the competition between attractive and repulsive regimes may lead to interesting structures when the system has been allowed to evolve for a long time.

In this paper, we retain the inter-agent force, but also equip 
agents with an additional stochastic motility mechanism. In certain cases, the stochastic motility mechanism can prevent complete collapse, even when the inter-agent force is always cohesive. For substantial parts of the discussion we keep $W(x)$ quite general, but to reveal some subtle aspects of the problem it is helpful to consider consider potentials of the form

$$
W(x)=\alpha|x|+\beta x^{2}+\gamma|x|^{3}+\delta x^{4},
$$

with the sign of the coefficient of the highest power present taken to be positive to ensure a cohesive interaction at large distances. When two coefficients on such a potential are nonzero and have opposite signs, a double-well potential will result and such cases are especially interesting. As the potential for a given force is indeterminate up to an additive constant, we may safely assume that $W(0)=0$ in all cases where the potential has a finite limit as the agent separation tends to zero.

The models to be considered are introduced in Section 2. In these models the cohesive force mechanism is always the same, but the stochastic motility mechanism can be classical linear diffusion, or more general linear processes, such as stable processes or fractional diffusion; and we also consider nonlinear diffusion. Some general results for the linear diffusion motility mechanism are given in Section 3. The motion of the mean (the centre of mass) is determined, and it is demonstrated that for a quadratic potential the variance has a finite asymptotic limit, motivating a study of equilibrium (that is, time-independent) solutions. It is shown that finite-mass equilibrium solutions are precluded if the potential is bounded, while decaying upper bounds on the tail of the equilibrium solution are exhibited for convex potentials. Specific examples of equilibrium solutions for linear diffusion involving algebraic potentials are addressed in Section 4. We note that broad questions concerning the longtime behaviour of systems such as those we study have been addressed elegantly using entropy methods and related techniques by Carrillo et al. [21], under assumptions of convexity of the potential. For more recent developments, see Bolley et al. [22]. However the approach that we follow here is based solely on analytical techniques with which most physicists and applied mathematicians would be familiar, and which prove to be especially useful for the potentials used on our specific illustrations.

To elucidate some subtle aspects of the diffusion-free evolution equation (1), we consider in Section 5 the solutions in the limit of small diffusivity of our model that incorporates linear diffusion. We show how, for the model potentials considered in Section 4, known results for Eq. (1) can be recovered. We also explore in Section 6 the effect of smoothing of a potential with singular behaviour at the origin on the structure of the equilibrium solutions and our analysis of the low-diffusivity limit leads to new insights concerning Eq. (1) for singular potentials.

In Section 7 we discuss equilibrium solutions when we take nonlinear diffusion as the stochastic motility mechanism and we establish conditions under which the equilibrium solution can have compact support. We return to the study of time evolving solutions in Section 8, where we consider a variety of linear motility mechanisms with the specific case of a quadratic cohesive potential. An interesting result is that for a broad class of processes related to fractional diffusion, the fate of the system is collapse of the probability density function to a single Dirac aggregate, that is, a single delta function.

For much of the paper, although a formal theorem-proof structure is not used, arguments presented are either completely rigorous, or involve completely written out calculations using standard analytical techniques and asymptotic methods. A little of the analysis in Section 5.1 and somewhat more of the analysis in Section 6 is more heuristic and speculative in nature and the reader's attention will be drawn to this at appropriate points.

The class of models that we consider can be generalized to arbitrary space dimension, and some of the arguments that we use can be adapted to return some exact results in dimensions greater than one. However, even where such extensions are possible, we have confined our attention to one space dimension for clarity in the discussion. It is perhaps appropriate to note that in the most mathematically subtle problems that we address in Sections 5 and 6, the difficulties in a one-dimensional analysis are already significant, and the scenario for which we present evidence in one dimension may be somewhat simpler than what happens in higher dimensional systems.

\section{Classes of models}

Many authors have investigated the behaviour of the nonlocal transport equation (1) with various interaction potentials $W$, where $f=-W^{\prime}$. For a purely attractive interaction the dynamics leads typically to the formation of a single Dirac aggregate either in infinite or in finite time depending on whether the interaction force $-W^{\prime}(x)$ tends to zero as $x \rightarrow 0$ or remains bounded away from zero as $x \rightarrow 0$ (like $W^{\prime}(x) \sim \operatorname{sgn}(x)$, corresponding to $W(x) \sim|x|)$; see [6] and the references therein.

The formation of pattern of multiple aggregates is known for double-well type repulsive-attractive interaction potentials, like for instance Morse potentials in swarming or models of cell aggregation, see e.g. [23, 24, 25, 26, 27, 28, 29].

In Fellner and Raoul $[5,6]$ the stability analysis of multiple aggregation pattern showed that the stable stationary states of Eq. (1) for smooth (i.e., $C^{2}$ ) double-well potential consist of finitely many Dirac masses. In contrast, a singular repulsive double-well potentials (like $W(x) \sim-|x|$ at $x=0$ ) will prevent the formation of Dirac mass and feature a continuous distribution of mass. In particular, an explicit example with a smoothed repulsive modulus potential at $x=$ (i.e. $W(x)=x^{2}-|x|_{\epsilon}$ where $|\cdot|_{\epsilon}$ is quadratically smoothed on $-\epsilon<x<\epsilon$ ) shows that Eq. (1) can have arbitrarily many $\left[O\left(\epsilon^{-1}\right)\right]$ Dirac masses as stable stationary states.

One result of the present paper shows that when a diffusion process (which we choose to be linear to enable explicit calculation) with a diffusivity that is sufficiently small relative to $\epsilon^{2}$ is added to Eq. (1) these multiple-aggregate stationary states will still exist as multiple smoothed aggregates, for which we construct the leading-order asymptotics.

The stochastic motility mechanisms that we consider fall into two broad classes. The first class (Section 2.1) covers 
classical linear diffusion [30, 31] and Lévy's stable processes [31], and also processes corresponding to fractional diffusion equations [32]. Classical linear diffusion and Levy's stable processes both arise as continuum limits of discrete-time random walk processes, with finite and infinite mean-square displacement per step, respectively. They are also relevant to continuoustime random walk processes, provided that the mean waiting time between steps is finite. Fractional diffusion equations are closely related to continuous-time random walk processes that cannot be decomposed into steps with finite mean-square displacement taking place at random intervals. Although in this paper we do not address simulations, it would be relatively straightforward to simulate the models with multiple random walkers moving on a lattice.

Our second class of motility models is nonlinear diffusion. A general rigorous stochastic processes basis for nonlinear diffusion appears to be lacking, but nonlinear diffusion processes naturally arise in mean-field treatments of systems with many random walkers who have local interactions such as volume exclusion, a preference for, or abhorrence of, near-neighbours, and so on [33]. Although in general the mean-field arguments do not produce rigorous continuum limits, the partial differential equations that they produce frequently agree with simulations [34, 35, 36, 37], and for the simplest exclusion processes some mean-field predictions turn out to agree with rigorous results $[38,39]$.

\subsection{Linear motility models}

To establish notation for later use, we define the Laplace transform and Fourier transform, respectively, by

$$
\begin{aligned}
& \widehat{f}(u)=\mathcal{L}\{f(t) ; t \mapsto u\}=\int_{0}^{\infty} e^{-u t} f(t) d t, \\
& \tilde{f}(q)=\mathcal{F}\{g(x) ; x \mapsto q\}=\int_{-\infty}^{\infty} e^{i q x} g(x) d x .
\end{aligned}
$$

For a function $h(x, t)$ of both position and time we shall use the spatial Fourier transform at fixed time $\tilde{h}(q, t)$, the temporal Laplace transform at fixed position $\widehat{h}(x, u)$ and the joint FourierLaplace transform

$$
\begin{aligned}
h^{*}(q, u) & =\mathcal{J}\{h(x, t) ; x \mapsto q, t \mapsto u\} \\
& =\int_{-\infty}^{\infty} e^{i q x} \int_{0}^{\infty} e^{-u t} h(x, t) d t d x \\
& =\mathcal{L}\{\tilde{h}(q, t) ; t \mapsto u\}=\mathcal{F}\{\widehat{h}(x, u) ; x \mapsto q\}
\end{aligned}
$$

Consider the evolution equation

$$
\frac{\partial \rho}{\partial t}=\mathcal{D} \rho(x, t)-\frac{\partial}{\partial x}\left\{\rho(x, t) \int_{-\infty}^{\infty} f(x-\xi) \rho(\xi, t) d \xi\right\},
$$

where $\mathcal{D}$ is a linear operator discussed in detail below, $f(x)=$ $-W^{\prime}(x)$ is an odd, locally integrable function and the integral over $(-\infty, \infty)$ is to be interpreted in a Cauchy principal value sense if it is not absolutely convergent, as

$$
f_{-\infty}^{\infty} f(x-\xi) \phi(\xi) d \xi=\lim _{L \rightarrow \infty} \int_{-L+x}^{L+x} f(x-\xi) \phi(\xi) d \xi .
$$

A consequence of this is that if we do not insist that solutions correspond to finite total mass, for most motility mechanisms combined with a symmetric potential, $\rho(x, t)=$ constant is a formal equilibrium solution for $x \in(-\infty, \infty)$.

We shall require solutions $\rho(x, t)$ of the equation to be nonnegative and, except for the discussion of rather subtle issues in Section 6.2, that the 'total mass' defined by Eq. (2) is finite and conserved. Subject only to modest decay requirements on $\rho(x, t)$ as $|x| \rightarrow \infty$ we see by integrating Eq. (9) that mass conservation requires

$$
\int_{-\infty}^{\infty} \mathcal{D} \rho(x, t) d x=\mathcal{F}\{\mathcal{D} \rho(x, t) ; q \mapsto 0\}=0 .
$$

We shall work with a normalised mass distribution and interpret

$$
p(x, t)=\frac{\rho(x, t)}{C}
$$

as a probability, so that

$$
\frac{\partial p}{\partial t}=\mathcal{D} p(x, t)-C \frac{\partial}{\partial x}\left\{p(x, t) \int_{-\infty}^{\infty} f(x-\xi) p(\xi, t) d \xi\right\} .
$$

Subject to the mass conservation condition, the linear operator $\mathcal{D}$ is one of the following (listed in order of increasing generality).

(a) A second-order differential operator in the space variable, for example, that corresponding to classical diffusion, with a constant diffusivity $D$ and a constant drift velocity $v$, so that

$$
\mathcal{D}=D \frac{\partial^{2}}{\partial x^{2}}-v \frac{\partial}{\partial x}
$$

(b) The infinitesimal generator of a Markov process on the real line or some other linear pseudodifferential operator operator acting on the space variable $x$, so that

$$
\mathcal{F}\{\mathcal{D} p(x, t) ; x \rightarrow q\}=-a(q) \tilde{p}(q, t),
$$

for example a symmetric stable process of order $\kappa \in(0,2]$ for which $a(q)=K|q|^{K}(K>0)$. In general, the mass conservation condition requires $a(0)=0$.

(c) A linear pseudodifferential operator acting on the space variable $x$ and the time variable $t$, so that

$$
\mathcal{J}\{\mathcal{D} p(x, t) ; x \rightarrow q, t \rightarrow u\}=-b(q, u) p^{*}(q, u),
$$

which will include processes equivalent to certain fractional diffusion equations. For mass conservation we need $b(0, u)=0$ for all $u>0$.

\subsection{Noninear motility models}

We may replace the motility mechanism of Section 2.1 with nonlinear diffusion:

$$
\frac{\partial \rho}{\partial t}=\frac{\partial}{\partial x}\left[\mathrm{D}(\rho) \frac{\partial \rho}{\partial x}\right]-\frac{\partial}{\partial x}\left\{\rho(x, t) f_{-\infty}^{\infty} f(x-\xi) \rho(\xi, t) d \xi\right\},
$$

Examples of the nonlinear diffusivity $\mathrm{D}(\rho)$ that arise from meanfield treatments of agent-based systems include a model allowing multiple agents per site for which $[40,41]$

$$
\mathrm{D}(\rho)=D_{0}\left\{g(\rho)+\rho g^{\prime}(\rho)\right\}
$$


with $g(\rho) \in[0,1]$; a model with excluded volume and a preferred local coordination number [35], for which

$$
\mathrm{D}(\rho)=D_{0}\left\{1-2 \rho(1-\rho) g^{\prime}(\rho) / g(\rho)\right\}
$$

with $g(\rho)>0$ and $\rho \in[0,1]$; and a variety of models related to chemotaxis [42].

We give some exact results for the power-law diffusivity $\mathrm{D}(\rho)=D_{\eta} \rho^{\eta}$ subject to the potentials $W(x)=\beta x^{2}$ and $W(x)=$ $\alpha|x|(\alpha, \beta>0)$ in Section 7 and Appendix C.

\section{Some general results for the linear diffusion case}

The explicit introduction of the Cauchy principal value in Eq. (13) guards against the possibility that the probability density function is so widely spread that

$$
\int_{-\infty}^{\infty}|W(x)| p(x, t) d x=\infty .
$$

Since the inter-agent force being cohesive at large distances opposes spreading, the integral can be interpreted in the ordinary sense in those cases where the stochastic motion by itself is known to produce densities that decay sufficiently rapidly at infinity. In the case where the stochastic component of the agent motion is classical diffusion, for an initial density of finite support, the density is exponentially small at remote distances for subsequent times, so that for potentials with at most polynomial growth, reference to the Cauchy principal value is unnecessary. Thus, if we take Eq. (14) to characterise the stochastic component of motion, we have

$$
\frac{\partial p}{\partial t}=\frac{\partial}{\partial x}\left[D \frac{\partial p}{\partial x}-v p(x, t)-C p(x, t) \int_{-\infty}^{\infty} f(x-\xi) p(\xi, t) d \xi\right] .
$$

\subsection{Time evolution of the mean and variance}

An evolution equation for the mean position

$$
\mu_{1}=\int_{-\infty}^{\infty} x p(x, t) d x
$$

(which corresponds to the centre of mass) can be obtained by multiplying Eq. (20) by $x$ and integrating. After an integration by parts, and assuming sufficient decay of $p(x, t)$ as $x \rightarrow \pm \infty$, we find that

$$
\frac{d \mu_{1}}{d t}=v+C I(t)
$$

where

$$
I(t)=\int_{-\infty}^{\infty} \int_{-\infty}^{\infty} f(x-\xi) p(x, t) p(\xi, t) d \xi d x .
$$

If we interchange the roles of $x$ and $\xi$ in the definition of $I(t)$, interchange the orders of integration, and use the oddness of $f$, we see that

$$
\begin{aligned}
I(t) & =\int_{-\infty}^{\infty} \int_{-\infty}^{\infty} f(x-\xi) p(x, t) p(\xi, t) d \xi d x \\
& =\int_{-\infty}^{\infty} \int_{-\infty}^{\infty} f(\xi-x) p(\xi, t) p(\xi, t) d x d \xi \\
& =\int_{-\infty}^{\infty} \int_{-\infty}^{\infty} f(\xi-x) p(x, t) p(\xi, t) d \xi d x \\
& =-\int_{-\infty}^{\infty} \int_{-\infty}^{\infty} f(x-\xi) p(x, t) p(\xi, t) d \xi d x=-I(t),
\end{aligned}
$$

so that $I(t) \equiv 0$, showing that the mean position $\mu_{1}$ moves with the mean drift velocity $v$ for the diffusive process, as one might reasonably have anticipated.

We now examine the time evolution of the variance

$$
\mathcal{V}=\int_{-\infty}^{\infty}\left(x-\mu_{1}\right)^{2} p(x, t) d x
$$

After two integrations by parts, and again assuming sufficient decay of $p(x, t)$ as $x \rightarrow \pm \infty$, we find that

$$
\frac{d \mathcal{V}}{d t}=2 D+2 C \int_{-\infty}^{\infty} \int_{-\infty}^{\infty}\left(x-\mu_{1}\right) f(x-\xi) p(x, t) p(\xi, t) d \xi d x
$$

In the absence of the inter-agent interaction, we recover the classical result that $\mathcal{V}(t)=\mathcal{V}(0)+2 D t$. Cohesive interactions would be expected to reduce the effective diffusion constant, but the effects can be somewhat more dramatic.

Consider the specific case of a quadratic cohesive potential

$$
W(x)=\beta x^{2},
$$

so that $f(x)=-2 \beta x$. We observe that for this potential,

$$
\begin{aligned}
\left(x-\mu_{1}\right) f(x-\xi) & =-2 \beta\left(x-\mu_{1}\right)(x-\xi) \\
& =-2 \beta\left[\left(x-\mu_{1}\right)^{2}+\left(x-\mu_{1}\right)\left(\mu_{1}-\xi\right)\right] .
\end{aligned}
$$

The integral in Eq. (26) can now be simplified and we find after a little algebra an ordinary differential equation for the variance:

$$
\mathcal{V}^{\prime}(t)+4 C \beta \mathcal{V}(t)=2 D
$$

Solving this equation in the usual way via an integrating factor, we find that

$$
\mathcal{V}(t)=\mathcal{V}(0) \exp (-4 C \beta t)+\frac{D}{2 C \beta}[1-\exp (-4 C \beta t)]
$$

Hence, independent of the details of the initial probability density function $P(x, 0)$ we find that for the quadratic potential (27),

$$
\lim _{t \rightarrow \infty} \mathcal{V}(t)=\frac{D}{2 C \beta}
$$

where the convergence is exponentially rapid. This example shows that it is possible for a cohesive potential to prevent ultimate escape to infinity via diffusion, but for the effect of diffusion to be strong enough to stop the mass distribution collapsing to a single delta function. 


\subsection{Equilibrium probability densities for general potentials}

In view of the result we have established for the time evolution of the mean, we may confine future attention in this section to the zero drift case $(v=0)$. Our proof that for a quadratic cohesive potential the variance converges to a positive constant as $t \rightarrow \infty$ suggests that one might seek steady-state solutions of Eq. (20), which should represent the long-time limiting state of the system. When $p(x, t)$ is independent of $t$, we shall write $p(x, t)=P(x)$.

A steady-state solution with zero flux requires the squarebracketed term in the right-hand side of Eq. (20) to be zero for all $x$. Thus

$$
P^{\prime}(x)=\frac{C P(x)}{D} \int_{-\infty}^{\infty} f(x-\xi) P(\xi) d \xi .
$$

Relevant solutions of Eq. (32) are subject to the constraints that

$$
P(x) \geq 0 \quad \text { and } \quad \int_{-\infty}^{\infty} P(x) d x=1 .
$$

Let $P(x)$ be a solution of Eq. (32) that satisfies the requirements (33) and let $\varpi(x)=P(x-c)$, for some constant $c$. Then on making the changes of variable $x \rightarrow x-c$ and $\xi \rightarrow \xi-c$ we find that

$$
-D \varpi^{\prime}(x)+\varpi(x) C \int_{-\infty}^{\infty} f(x-\xi) \varpi(\xi) d \xi=0 .
$$

Hence if $P(x)$ is a solution of our problem, so is $p(x-c)$. We have seen that for the time-dependent problem, the mean $\mu_{1}$ is constant, so the existence of a free parameter in the general solution for a steady state, which we hope captures the longtime limit, is to be expected. We are at liberty to set $\mu_{1}=0$ if we wish, though we shall not always do this.

Does the oddness of the function $f$ ensure that $P$ is symmetric about its mean, that is, $P\left(x-\mu_{1}\right) \equiv P\left(\mu_{1}-x\right)$ ? If again we take $P$ to be a solution and we now let $\varphi(x)=P(c-x)$, then we have

$$
\begin{aligned}
-D \varphi^{\prime}(x)+C \varphi(x) \int_{-\infty}^{\infty} f(x-\xi) \varphi(\xi) d \xi \\
=D P^{\prime}(c-x)+C P(c-x) \int_{-\infty}^{\infty} f(x-\xi) P(c-\xi) d \xi \\
=D P^{\prime}(c-x)+C P(c-x) \int_{-\infty}^{\infty} f(x-c+\zeta) P(\zeta) d \zeta \\
=D P^{\prime}(c-x)-C P(c-x) \int_{-\infty}^{\infty} f(c-x-\zeta) P(\zeta) d \zeta=0 .
\end{aligned}
$$

Hence if $P(x)$ is a solution, so is $P(c-x)$. In particular, if $P_{0}(x)$ is a solution with mean zero, then $P_{+}(x)=P_{0}\left(x-\mu_{1}\right)$ and $P_{-}(x)=P_{0}\left(\mu_{1}-x\right)$ are both solutions of the problem with the same mean $\mu_{1}$, and a common value at $x=\mu_{1}$. This supports, but does not prove conclusively, the claim that $P_{0}\left(x-\mu_{1}\right) \equiv$ $P_{0}\left(\mu_{1}-x\right)$. Of course, if $p(x, 0)$ is symmetric about $x=\mu_{1}$ and $P(x)=\lim _{t \rightarrow \infty} p(x, t)$ exists, then because of the symmetries of the problem, $P(x)$ is necessarily also symmetric about the mean.
If we eliminate $f$ in favour of the derivative of the potential $\left[f(x-\xi)=-W^{\prime}(x-\xi)\right]$ then we have

$$
P^{\prime}(x)=-\frac{C P(x)}{D} \int_{-\infty}^{\infty} W^{\prime}(x-\xi) P(\xi) d \xi
$$

and so

$$
\frac{d}{d x} \log [P(x)]=-\frac{C}{D} \int_{-\infty}^{\infty} W^{\prime}(x-\xi) P(\xi) d \xi,
$$

corresponding to

$$
P(x)=\text { constant } \times \exp \left\{-\frac{C}{D} \int_{-\infty}^{\infty} W(x-\xi) P(\xi) d \xi\right\}
$$

From the requirements (33), the constant must be strictly positive and so we see that unless $W(x) \rightarrow \infty$ at finite $x$, we cannot have $P(x)=0$ anywhere-for a continuous potential, equilibrium solutions with bounded support are precluded. Hence in particular $P\left(\mu_{1}\right) \neq 0$ and

$$
P(x)=P\left(\mu_{1}\right) \exp \left\{-\frac{C}{D} \int_{-\infty}^{\infty}\left[W(x-\xi)-W\left(\mu_{1}-\xi\right)\right] P(\xi) d \xi\right\} .
$$

If $W(z)$ is bounded, that is, $|W(z)|<w<\infty$ for all $z$, then from the triangle inequality $-2 w \leq\left|W(x-\xi)-W\left(\mu_{1}-\xi\right)\right| \leq 2 w$ and so, recalling also the normalization integral (33), we deduce the bounds

$$
P\left(\mu_{1}\right) \exp \left(-\frac{2 w C}{D}\right)<P(x)<P\left(\mu_{1}\right) \exp \left(\frac{2 w C}{D}\right) .
$$

The lower bound for $P(x)$ is inconsistent with the requirement that $p(x)$ be integrable on $(-\infty, \infty)$. There are therefore no "finite mass" equilibrium solutions of the problem on $(-\infty, \infty)$ for a bounded potential.

Suppose now that $W(z)$ is a convex function (i.e., is "concave up") and that

$$
-\infty<\int_{-\infty}^{\infty} W(x-\xi) P(\xi) d \xi<\infty,
$$

an innocent assumption for potentials of at most polynomial growth at infinity. Interpreting the integral as an expectation and using Jensen's inequality $\mathbb{E}\{\phi(X)\} \geq \phi(\mathbb{E}\{X\})$ for convex $\phi$, we have

$$
\int_{-\infty}^{\infty} W(x-\xi) P(\xi) d \xi \geq W\left(x-\mu_{1}\right)
$$

and so

$$
\frac{P(x)}{P\left(\mu_{1}\right)} \leq \text { constant } \times \exp \left(-\frac{C}{D} W\left(x-\mu_{1}\right)\right) .
$$

Thus if we want a non-trivial equilibrium solution that decays algebraically at infinity rather than exponentially, we need rather slow growth of the potential $W(x)$ as $x \rightarrow \infty$, for example, $W(x) \sim$ constant $\times \log (x)$.

\section{Equilibrium linear diffusion with algebraic potentials}

We address here equilibrium solutions for special cases of the class of potentials

$$
W(x)=\alpha|x|+\beta x^{2}+\gamma|x|^{3}+\delta x^{4},
$$




\subsection{An autonomous system analysis}

We shall assume that the even potential function $W(x)$ is given by

$$
W(x)=\alpha|x|+\beta x^{2},
$$

where either (i) $\alpha>0$ and $\beta=0$ or (ii) $\beta>0$, to ensure global attraction. Then we have

$$
W^{\prime}(x)=\alpha \operatorname{sgn}(x)+2 \beta z, \quad W^{\prime \prime}(x)=2 \alpha \delta(x)+2 \beta
$$

where we denote Dirac's delta function by $\delta(\cdot)$ to distinguish it from the parameter $\delta$ used elsewhere in this paper, and so

$$
\begin{aligned}
\frac{d^{2}}{d x^{2}} \log [P(x)] & =-\frac{C}{D} \int_{-\infty}^{\infty} W^{\prime \prime}(x-\xi) P(\xi) d \xi \\
& =-\frac{C}{D} \int_{-\infty}^{\infty}[2 \alpha \delta(x-\xi)+2 \beta] P(\xi) d \xi
\end{aligned}
$$

Hence from Eq. (34),

$$
\frac{P^{\prime \prime}(x)}{P(x)}-\frac{P^{\prime}(x)^{2}}{P(x)^{2}}=-\frac{2 \alpha C}{D} P(x)-\frac{2 \beta C}{D} .
$$

This is an autonomous differential equation and can be analysed in the standard manner. We note that there is related earlier work for Morse-type potentials [18].

We observe that if $\varpi(x)$ is a solution of Eq. (42), then so are $\varpi\left(x-x_{0}\right)$ and $\varpi\left(x_{0}-x\right)$ for any constant $x_{0}$. Choose $x_{0}$ to be a point where $\varpi^{\prime}\left(x_{0}\right)=0$. Then $\varpi\left(x-x_{0}\right)$ and $\varpi\left(x_{0}-x\right)$ are both solutions of the initial value problem defined by Eq. (42) with $p\left(x_{0}\right)=\varpi\left(x_{0}\right)$ and $p^{\prime}\left(x_{0}\right)=0$. Hence from the uniqueness of solutions to the initial value problem, $\varpi\left(x-x_{0}\right)=\varpi\left(x_{0}-x\right)$ for all $x$. Thus we see that every solution of Eq. (42) has a reflection symmetry around any stationary point. This forces the solution to be unimodal, as otherwise there would be infinitely many maxima and minima, with all maxima having the same height, and decay of $p$ at $\pm \infty$, required for normalisation, would be precluded. From the symmetry we conclude that the unique stationary point corresponds to the mean $\mu_{1}$.

If we let $Q=P^{\prime}(x)$ we obtain

$$
\frac{1}{2 P} \frac{d}{d P}\left(Q^{2}\right)-\frac{Q^{2}}{P^{2}}=-\frac{2 \alpha C}{D} P-\frac{2 \beta C}{D}
$$

and multiplying both sides by $2 / P$ we see that

$$
\frac{d}{d P}\left(\frac{Q^{2}}{P^{2}}\right)=-\frac{4 \alpha C}{D}-\frac{4 \beta C}{D P}
$$

We seek a solution with $P^{\prime}\left(\mu_{1}\right)=0$, corresponding to $Q=0$ at $P=P\left(\mu_{1}\right)$ and $P(x)$ monotonic decaying for $x>\mu_{1}$, so that

$$
-\frac{Q^{2}}{P^{2}}=-\frac{4 \alpha C}{D}\left[P\left(\mu_{1}\right)-P\right]-\frac{4 \beta C}{D} \log \left[\frac{P\left(\mu_{1}\right)}{P}\right] .
$$

Hence

$$
P^{\prime}(x)^{2}=\frac{4 \alpha C}{D} P(x)^{2}\left[P\left(\mu_{1}\right)-P(x)\right]+\frac{4 \beta C P(x)^{2}}{D} \log \left[\frac{P\left(\mu_{1}\right)}{P(x)}\right] .
$$

In general Eq. (43) has to be solved numerically, with an iterative procedure to determine $P\left(\mu_{1}\right)$ based on the normalisation condition

$$
\int_{-\infty}^{\infty} P(x) d x=1
$$

Case $\alpha=0$. If $\alpha=0$ and we write $P(x)=P\left(\mu_{1}\right) \exp [-\psi(x)]$ with $\psi\left(\mu_{1}\right)=0$ and $\psi(x)$ increasing for $x>\mu_{1}$, we have

$$
\psi^{\prime}(x)^{2}=\frac{4 \beta C \psi(x)}{D}, \quad \text { giving } \quad \frac{d x}{d \psi}=\frac{D^{1 / 2}}{2 \beta^{1 / 2} C^{1 / 2} \psi^{1 / 2}}
$$

and the unique solution with $\psi\left(\mu_{1}\right)=0$ is given by

$$
x-\mu_{1}=D^{1 / 2}(\beta C)^{-1 / 2} \psi(x)^{1 / 2},
$$

that is,

$$
\psi(x)=\frac{\beta C\left(x-\mu_{1}\right)^{2}}{D} .
$$

Fixing $P\left(\mu_{1}\right)$ using the normalisation condition (44), we obtain the solution

$$
P(x)=\left(\frac{\beta C}{D}\right)^{1 / 2} \exp \left\{-\frac{\beta C\left(x-\mu_{1}\right)^{2}}{D}\right\} .
$$

We note that the upper bound (37) is sharp.

Case $\beta=0$. If $\beta=0$, then to have a cohesive potential at large separations we require $\alpha>0$, and we have

$$
P^{\prime}(x)^{2}=\frac{4 \alpha C}{D} P^{2}\left[P\left(\mu_{1}\right)-P\right] .
$$

If we write $P(x)=P\left(\mu_{1}\right)\left[1-\chi\left(x-\mu_{1}\right)\right]^{2}$ with $\chi(0)=0, \chi^{\prime}(z)>0$ for $z>0$ and $\chi(z) \rightarrow 1$ as $z \rightarrow \infty$ then a simple differential equation for $\chi$ results, and we find that the unique equilibrium solution is

$$
P(x)=\frac{\alpha C}{4 D}\left\{1-\tanh ^{2}\left[\frac{\alpha C\left(x-\mu_{1}\right)}{2 D}\right]\right\}=\frac{\alpha C}{4 D} \operatorname{sech}^{2}\left[\frac{\alpha C\left(x-\mu_{1}\right)}{2 D}\right] .
$$

Since $\operatorname{sech}^{2}(z) \sim 4 \exp (-2|z|)$ as $z \rightarrow \pm \infty$, we have

$$
P(x) \sim \frac{\alpha C}{D} \exp \left(-\frac{\alpha C\left|x-\mu_{1}\right|}{D}\right) \text { as }\left|x-\mu_{1}\right| \rightarrow \infty .
$$

Once again we find that the bound (37) is quite sharp.

Case $\alpha \neq 0, \beta>0$. To facilitate numerical solutions and perturbation analysis, we scale the problem by writing

$$
X=\left(\frac{C \beta}{D}\right)^{1 / 2}\left(x-\mu_{1}\right), \quad \epsilon=\frac{\alpha P\left(\mu_{1}\right)}{\beta} \neq 0,
$$

where $\epsilon$ need not be small in magnitude, and may be positive or negative. We seek a solution of the form

$$
P(x)=P\left(\mu_{1}\right) \exp [-\Psi(X)], \quad \Psi(0)=0 .
$$

The normalisation condition becomes

$$
\left(\frac{D}{C \beta}\right)^{1 / 2} P\left(\mu_{1}\right) \int_{-\infty}^{\infty} \exp [-\Psi(X)] d X=1,
$$

while the differential equation to be solved reduces to

$$
\Psi^{\prime}(X)^{2}=4 \Psi(X)+4 \epsilon\{1-\exp [-\Psi(X)]\} .
$$


We remark that $C, D, \alpha$ and $\beta$ are the physical parameters: the parameter $\epsilon$ contains $P\left(\mu_{1}\right)$. Since $\Psi(X) \rightarrow 0$ as $X \rightarrow 0$, we see that in the vicinity of the origin,

$$
\Psi^{\prime}(X)^{2} \sim 4(1+\epsilon) \Psi(X),
$$

so that we shall always have $\epsilon>-1$. Hence, if $\alpha<0$ we have

$$
P\left(\mu_{1}\right)<\frac{\beta}{|\alpha|} \quad \text { if } \beta>0 \text { and } \alpha<0 .
$$

Since $\Psi(X)$ is an even function of $X$, we naturally seek a small- $X$ expansion in integer powers of $X^{2}$. Using the functions SeriesCoefficient and Solve in Mathematica (Wolfram Research, Version 8.0.0.0) we easily generate the expansion

$$
\begin{aligned}
\Psi(X)= & (1+\epsilon) X^{2}-\frac{\epsilon(1+\epsilon)}{6} X^{4}+\frac{\epsilon(1+\epsilon)(3+4 \epsilon)}{90} X^{6} \\
& -\frac{\epsilon(1+\epsilon)\left(15+48 \epsilon+34 \epsilon^{2}\right)}{2520} X^{8} \\
& +\frac{\epsilon(1+\epsilon)\left(105+624 \epsilon+1014 \epsilon^{2}+496 \epsilon^{3}\right)}{113400} X^{10} \\
& +O\left(X^{12}\right) .
\end{aligned}
$$

We also note that as $X \rightarrow \infty$ we have $\Psi^{\prime}(X) \sim 2 \Psi(X)^{1 / 2}$, from which it follows that $\Psi(X) \sim X^{2}$ as $X \rightarrow \infty$ for all $\epsilon>-1$. In the degenerate case $\epsilon=0$ we have $\Psi(X) \equiv X^{2}$ for all $X$. From the leading-order large $X$ asymptotic behaviour of $\Psi(X)$, we see that the exponential term in Eq. (51) can be neglected to all orders in a large- $X$ asymptotic expansion in powers of $X$, and it is straightforward to verify that

$$
\Psi(X)=[X+b(\epsilon)]^{2}-\epsilon+O\left(X^{-2}\right) \text { as } X \rightarrow \infty,
$$

where $b(\epsilon)$ is not determined by the asymptotic analysis, reflects the initial condition $\Psi(0)=0$, and if required needs to be determined by a matching procedure. In practice, to obtain solutions to adequate precision for plotting one may use the series expansion (54) for $0 \leq X \leq X_{0}$ and the direct numerical solution of the ODE for $X \geq X_{0}$, using the series estimate of $\Psi\left(X_{0}\right)$ as the initial value. The choice of $X_{0}$ and the optimal number of terms retained in the expansion (54) depends on $\epsilon$.

Equilibrium solutions $P(x)$ for a range of values of the dimensionless parameter $\alpha \sqrt{C /(\beta D)}$ are shown in Fig. 1. We plot $(C \beta / D)^{1 / 2} P$ as a function $X=(C \beta / D)^{1 / 2}\left(x-\mu_{1}\right)$. To obtain these solutions we have solved the ODE for $\Psi$ for a number of positive and negative values of $\epsilon$. For a given value of $\epsilon$ we can infer $(C \beta / D)^{1 / 2} P\left(\mu_{1}\right)$ and $\alpha \sqrt{C /(\beta D)}$ by noting that Eq. (50) can be written as

$$
\frac{\epsilon}{\alpha \sqrt{C /(\beta D)}}=\left(\frac{D}{C \beta}\right)^{1 / 2} P\left(\mu_{1}\right)=\left\{\int_{0}^{\infty} \frac{e^{-\Psi} d \Psi}{\sqrt{\Psi+\epsilon\left(1-e^{-\Psi}\right)}}\right\}^{-1} .
$$

The analysis of this problem for very small $D$ is quite subtle and is discussed in Section 5.1.

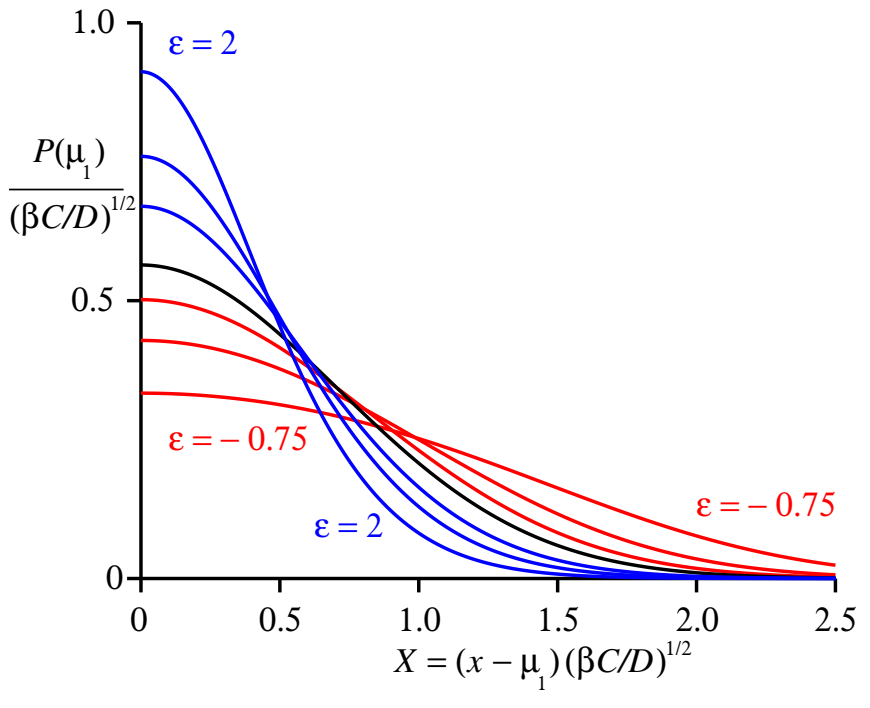

Figure 1: (Color online) For the potential $W(x)=\alpha|x|+\beta x^{2}$, we show the scaled equilibrium solution $(C \beta / D)^{-1 / 2} P(x)=(C \beta / D)^{-1 / 2} P\left(\mu_{1}\right) \exp [-\Psi(X)]$, where $X=(C \beta / D)^{1 / 2}\left(x-\mu_{1}\right)$. The values of $\epsilon$ chosen, listed in order of increasing height at the origin, are $-0.75,-0.5,-0.25$, shown in red; 0 , shown in black; $0.5,1,2$, shown in blue. Corresponding values of $\alpha(C /(\beta D))^{1 / 2}$ are -2.248 , $1.167,-0.498,0,0.746,1.316,2.193$

\subsection{Analysis based on integrals}

We now consider the case

$$
W(x)=\beta x^{2}+\delta x^{4},
$$

with $\delta>0$. If we introduce the $k$ th central moment of the density,

$$
\mu_{k}^{(\mathrm{c})}=\int_{-\infty}^{\infty}\left(x-\mu_{1}\right)^{k} P(x) d x, \quad k \in \mathbb{N}
$$

and note that $\mu_{1}^{(\mathrm{c})}=0$, then we easily show that

$$
\begin{aligned}
\int_{-\infty}^{\infty} & {\left[W(x-\xi)-W\left(\mu_{1}-\xi\right)\right] P(\xi) d \xi } \\
= & \int_{-\infty}^{\infty}\left[W\left(x-\mu_{1}-\mu_{1}-\xi\right)-W\left(\mu_{1}-\xi\right)\right] P(\xi) d \xi \\
= & \delta\left(x-\mu_{1}\right)^{4}+\left(6 \mu_{2}^{(\mathrm{c})} \delta+\beta\right)\left(x-\mu_{1}\right)^{2}-4 \delta \mu_{3}^{(\mathrm{c})}\left(x-\mu_{1}\right),
\end{aligned}
$$

giving

$$
\begin{gathered}
P(x)=P\left(\mu_{1}\right) \exp \left\{-\frac{C}{D}\left[\delta\left(x-\mu_{1}\right)^{4}+\left(6 \mu_{2}^{(\mathrm{c})} \delta+\beta\right)\left(x-\mu_{1}\right)^{2}\right.\right. \\
\left.\left.-4 \delta \mu_{3}^{(\mathrm{c})}\left(x-\mu_{1}\right)\right]\right\} .
\end{gathered}
$$

Thus the form of $P(x)$ has been largely determined, although it remains to check consistency of the central moments that have appeared in Eq. (60). If we observe that

$$
\frac{P\left(\mu_{1}+s\right)}{P\left(\mu_{1}-s\right)}=\exp \left(-8 \delta \mu_{3}^{(\mathrm{c})} s\right)
$$

is strictly greater than 1 for all $s>0$ if $\mu_{3}^{(\mathrm{c})}<0$ and strictly less than 1 for all $s>0$ if $\mu_{3}^{\text {(c) }}>0$ we see that the requirement that 
$\mu_{1}^{(\mathrm{c})}=0$, which corresponds to

$$
\int_{0}^{\infty} s P\left(\mu_{1}+s\right) d s=\int_{0}^{\infty} s P\left(\mu_{1}-s\right) d s,
$$

forces us to conclude that $\mu_{3}^{\text {(c) }}=0$. Thus we have shown that

$$
P(x)=P\left(\mu_{1}\right) \exp \left\{-\frac{C}{D}\left[\delta\left(x-\mu_{1}\right)^{4}+\left(6 \mu_{2}^{(\mathrm{c})} \delta+\beta\right)\left(x-\mu_{1}\right)^{2}\right]\right\},
$$

where we require

$$
\begin{gathered}
1=P\left(\mu_{1}\right) \int_{-\infty}^{\infty} \exp \left\{-\frac{C}{D}\left[\delta s^{4}+\left(6 \mu_{2}^{(\mathrm{c})} \delta+\beta\right) s^{2}\right]\right\} d s, \\
\mu_{2}^{(\mathrm{c})}=P\left(\mu_{1}\right) \int_{-\infty}^{\infty} s^{2} \exp \left\{-\frac{C}{D}\left[\delta s^{4}+\left(6 \mu_{2}^{(\mathrm{c})} \delta+\beta\right) s^{2}\right]\right\} d s .
\end{gathered}
$$

Dividing these equations yields a single transcendental equation for $\mu_{2}^{(\mathrm{c})}$. where

The density $P(x)$ prescribed by Eq. (61) has stationary points

$$
\begin{aligned}
4 \delta(x- & \left.\mu_{1}\right)^{3}+2\left(\beta+6 \mu_{2}^{(\mathrm{c})} \delta\right)\left(x-\mu_{1}\right) \\
& =4 \delta\left(x-\mu_{1}\right)\left[\left(x-\mu_{1}\right)^{2}+\frac{\beta+6 \mu_{2}^{(\mathrm{c})} \delta}{2 \delta}\right]=0 .
\end{aligned}
$$

If $\beta \geq-6 \mu_{2}^{(\mathrm{c})} \delta$ the density $P(x)$ is unimodal, with its peak at the mean, while if $\beta<-6 \mu_{2}^{(\mathrm{c})} \delta$ it is bimodal, with peaks at

$$
x=\mu_{1} \pm \varpi, \quad \text { where } \quad \varpi=\sqrt{\left|\beta+6 \mu_{2}^{(\mathrm{c})} \delta\right| /(2 \delta)} .
$$

Note that the condition for $P(x)$ to be bimodal is more restrictive than the condition $\beta<0$ needed for the potential $W(x)$ to have two minima arranged symmetrically around the mean, and the distance between the peaks of $P(x)$, when there are peaks, is not equal to the distance between the potential minima. At the "critical point" where $\beta=-6 \mu_{2}^{(\mathrm{c})} \delta$, the integrals in Eqs (62) and (63) can be evaluated exactly in terms of the usual gamma function and we find that the criticality condition is

$$
\beta+6 \frac{\Gamma(3 / 4)}{\Gamma(1 / 4)}\left(\frac{D \delta}{C}\right)^{1 / 2}=0, \text { where } 6 \frac{\Gamma(3 / 4)}{\Gamma(1 / 4)} \approx 2.0279,
$$

so the equilibrium density is bimodal if $\beta<-2.0279(D \delta / C)^{1 / 2}$.

For numerical analysis it is convenient to write

$$
\begin{aligned}
M & =\mu_{2}^{(\mathrm{c})} \sqrt{\frac{C \delta}{D}} \quad \theta=\beta \sqrt{\frac{C}{D \delta}}, \\
\Pi(X) & =\left(\frac{D}{C \delta}\right)^{1 / 4} P\left(\mu_{1}+\left(\frac{D}{C \delta}\right)^{1 / 4} X\right) .
\end{aligned}
$$

From Eqs (61), (62) and (63) we have

$$
\begin{aligned}
& \Pi(X)=\Pi(0) \exp \left[-X^{4}-(6 M+\theta) X^{2}\right], \\
& \frac{1}{\Pi(0)}=2 \int_{0}^{\infty} \exp \left[-X^{4}-(6 M+\theta) X^{2}\right] d X, \\
& \frac{M}{\Pi(0)}=2 \int_{0}^{\infty} X^{2} \exp \left[-X^{4}-(6 M+\theta) X^{2}\right] d X .
\end{aligned}
$$

The integrals are not elementary, but can be evaluated in terms of the parabolic cylinder function $D_{v}(z)$ available in MathematICA using the equations [47]

$$
\begin{aligned}
D_{v}(z) & =U\left(-\frac{1}{2}-v, z\right) \\
U(a, z) & =\frac{\exp \left(-z^{2} / 4\right)}{\Gamma\left(\frac{1}{2}+a\right)} \int_{0}^{\infty} t^{a-1 / 2} \exp \left(-\frac{1}{2} t^{2}-z t\right) d t
\end{aligned}
$$

We find that

$$
\Pi(0)=\frac{2^{1 / 4}}{\pi^{1 / 2}} \frac{\exp \left[(6 M+\theta)^{2} / 8\right]}{D_{-1 / 2}\left(2^{-1 / 2}(6 M+\theta)\right)},
$$

where $M$ satisfies the equation

$$
M=\frac{D_{-3 / 2}\left(2^{-1 / 2}(6 M+\theta)\right)}{2 \sqrt{2} D_{-1 / 2}\left(2^{-1 / 2}(6 M+\theta)\right)} .
$$

One would expect that as $\theta \rightarrow-\infty$, the scaled variance $M$ should diverge to infinity. Using the known asymptotic form of the parabolic cylinder function $D_{v}(z)$ for $z \rightarrow \infty$ and for $z \rightarrow-\infty$ to explore the possible scenarios $6 M+\theta \rightarrow \infty$ and $6 M+\theta \rightarrow-\infty$ we find that it is the latter that occurs, and it is easy to show that

$$
M \approx-\theta / 8+1 /(2 \theta) \text { when } \theta \ll-1 .
$$

The behaviour of $M$ for $\theta \gg 1$ can also be deduced from the asymptotic form of $D_{v}(z)$ as $z \rightarrow \infty$ and we find that

$$
M \approx 2^{-1} \theta^{-2} \text { when } \theta \gg 1 .
$$

We show the scaled variance $M$ as a function of $\theta$ in Fig. 2: the numerical solution of Eq. (74), shown as a solid black curve, is well represented by the $\theta \ll-1$ approximation (75), shown as a broken blue curve, when $\theta<-7$, and also well represented by the $\theta \gg 1$ approximation (75), shown as a broken red curve, when $\theta>7$.

We show the scaled equilibrium probability density function $\Pi(X)$ for several values of $\theta$ in Fig. 3 .

\section{Equilibrium linear diffusion in the low-diffusivity limit}

It is of interest to consider the limiting behaviour of our equilibrium solutions under linear diffusion when $D \rightarrow 0$, especially as it may elucidate the behaviour of equilibrium solutions of the diffusion-free evolution equation (1).

\subsection{The potential $W(x)=\alpha|x|+\beta x^{2}$}

From the exact solutions (45) and (46) for the cases $\alpha=0$ (and so $\beta>0$ ) and $\beta=0$ (and so $\alpha>0$ ), respectively, we see that as $D \rightarrow 0$, the distribution becomes a sharp peak centred on the mean $\mu_{1}$. The limiting distribution is $P(x)=\delta\left(x-\mu_{1}\right)$. Clearly, then, if $\alpha>0$ and $\beta>0$, collapse to a delta function must also occur.

The case $\alpha<0$ and $\beta>0$, to which we now confine our attention, is more interesting. This case is the simplest doublewell potential with competition between attraction and repulsion that can be used with the deterministic equation (1) and 


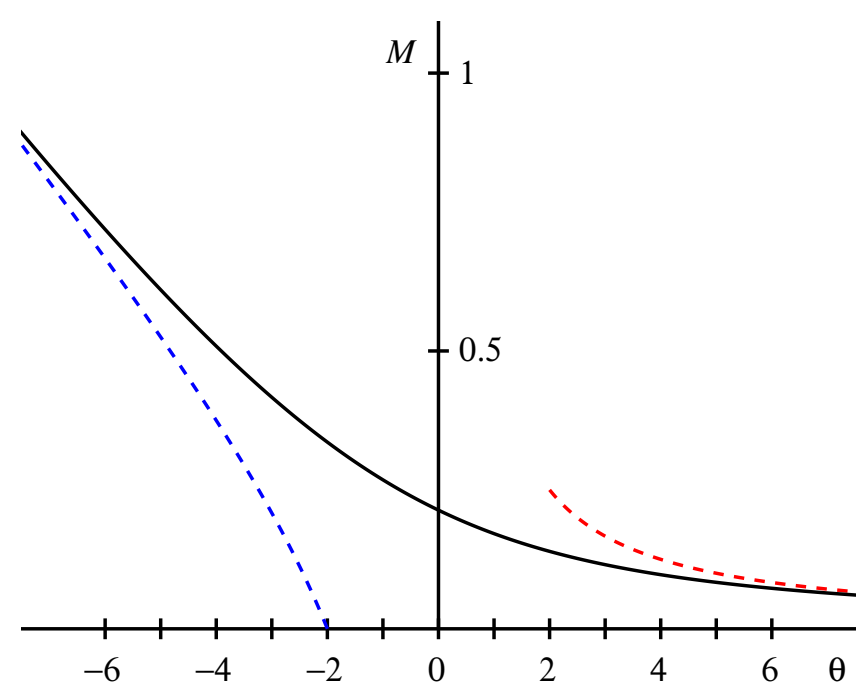

Figure 2: (Color online) The scaled variance $M=\mu_{2}^{(\mathrm{c})} \sqrt{C \delta / D}$ of the equilibrium probability density function for the potential $W(x)=\beta x^{2}+\delta x^{4}$ as a function of the parameter $\theta=\beta \sqrt{C /(D \delta)}$ : the solid black curve comes from numerical solution of the transcendental equation (74); broken curves show the asymptotic solutions for $\theta \ll-1$ (blue) and $\theta \gg 1$ (red). The equilibrium density is is bimodal when $\theta<-2.0279$ (see Fig. 3).

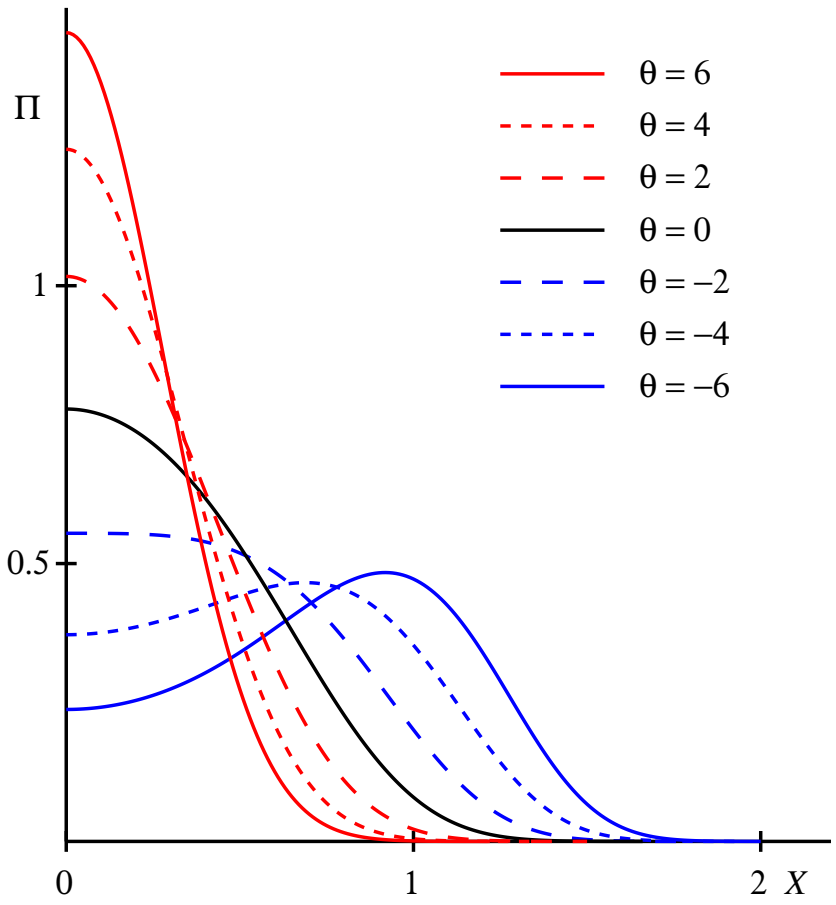

Figure 3: (Color online) The scaled equilibrium probability density function $\Pi(X)$ for the potential $W(x)=\beta x^{2}+\delta x^{4}$ as a function of the parameter $\theta=$ $\beta \sqrt{C /(D \delta)}$, determined by numerical solution of the transcendental equation (74) and use of Eqs (68) and (73) our ability to obtain a number of exact analytic results when a linear diffusion mechanism is present opens the possibility of using the small-diffusivity limit to shed light on the behaviour of Eq. (1). Consider the purely non-local interaction equation (1), that is, diffusion has been completely removed. For a system of finite mass $C$ the normalized equilibrium distribution $P$ with mean $\mu_{1}$ can be determined very simply as follows. Since

$$
\int_{\infty}^{\infty} 2 \beta(x-\xi) P(\xi) d \xi=2 \beta\left(x-\mu_{1}\right)
$$

the harmonic confining interaction potential $\beta x^{2}$ acts like an external confining potential $\beta x^{2}$ and our equilibrium equation becomes

$$
P(x) \int_{-\infty}^{\infty}\left[2 \beta\left(x-\mu_{1}\right)-|\alpha| \int_{-\infty}^{\infty} \operatorname{sgn}(x-\xi) P(\xi) d \xi\right]=0 .
$$

From this is follows that either $P(x)=0$ or

$$
2|\alpha| \int_{-\infty}^{x} P(\xi) d \xi-1=2 \beta\left(x-\mu_{1}\right) .
$$

In the latter case $P(x)=\beta /|\alpha|$ and so the only finite-mass equilibrium solution with mean $\mu_{1}$ is

$$
P(x)= \begin{cases}\beta /|\alpha|, & \left|x-\mu_{1}\right|<|\alpha| /(2 \beta), \\ 0 & \left|x-\mu_{1}\right|>|\alpha| /(2 \beta) .\end{cases}
$$

We now turn on diffusion. We know from Section 4.1 that the distribution is unimodal, but that when $\alpha<0$ and $\beta>0$ we have the bound $P\left(\mu_{1}\right)<\beta /|\alpha|$ [Eq. 53)]. Hence in this case the limiting distribution of $P(x)$ as $D \rightarrow 0$ is not a delta function: the limiting distribution is of non-zero width. Because of the symmetry of the solution about $x=\mu_{1}$ and the monotonic decay of $P(x)$ for $x>\mu_{1}$, we can write the normalization condition as

$$
\begin{aligned}
\frac{1}{2} & =\int_{0}^{\infty} P(x) d x=\int_{0}^{P\left(\mu_{1}\right)} P\left|\frac{d x}{d P}\right| d P \\
& =\int_{0}^{P\left(\mu_{1}\right)} \frac{P d P}{\left\{\frac{4 \beta C P^{2}}{D} \log \left[\frac{P\left(\mu_{1}\right)}{P}\right]-\frac{4|\alpha| C}{D} P^{2}\left[P\left(\mu_{1}\right)-P\right]\right\}^{1 / 2}} .
\end{aligned}
$$

We have eliminated $P^{\prime}(x)$ ising Eq. (43). If we write $P=$ $P\left(\mu_{1}\right) p$ we obtain

$$
1=\frac{P\left(\mu_{1}\right) D^{1 / 2}}{(|\alpha| C)^{1 / 2}} \int_{0}^{1} \frac{d p}{\left\{\frac{\beta}{|\alpha|} \log \left[\frac{1}{p}\right]-P\left(\mu_{1}\right)(1-p)\right\}^{1 / 2}} .
$$

In order to retain normalization, it is necessary that the integral diverges in an appropriate manner as $D \rightarrow 0$ and this requires $P\left(\mu_{1}\right) \rightarrow \beta /|\alpha|$ in a special way. The details of the analysis will be found in Appendix A. We find that, where $K$ is positive and independent of $D$,

$$
\frac{\beta}{|\alpha|}-P\left(\mu_{1}\right) \sim K \exp \left[-\left(\frac{C \alpha^{2}}{2 \beta D}\right)^{1 / 2}\right]
$$


giving rapid convergence of $P\left(\mu_{1}\right)$ to $\beta /|\alpha|$. From Eq. (42) we have

$$
\frac{P^{\prime \prime}\left(\mu_{1}\right)}{P\left(\mu_{1}\right)}=-\frac{2|\alpha| C}{D}\left(\frac{\beta}{|\alpha|}-P\left(\mu_{1}\right)\right) \sim-\frac{2|\alpha| C K}{D} \exp \left[-\left(\frac{C \alpha^{2}}{2 \beta D}\right)^{1 / 2}\right] .
$$

Thus we see $P^{\prime \prime}\left(\mu_{1}\right) \rightarrow 0$ very rapidly as $D \rightarrow 0$ and so for very small $D$, there is an extended region in the neighbourhood of $x=\mu_{1}$ in which $P(x) \approx \beta /|\alpha|$. This result strongly suggests that $P(x) \rightarrow \beta /|\alpha|$ as $D \rightarrow 0$ in a finite interval surrounding the origin.

If we look again at Eq. (43) we see that it is satisfied on finite intervals by $P(x)=0$ or by $P(x)=P\left(\mu_{1}\right)$ and we conclude that for small $D$ we have $P(x) \approx \beta /|\alpha|$ for $\left|x-\mu_{1}\right|<L-\epsilon$ and $P(x) \approx 0$ for $\left|x-\mu_{1}\right|<L-\epsilon$, with a transition zone of width $\approx 2 \epsilon$ separating these regions. Experience with singular perturbation problems suggests that the transition zone should be a narrow zone of rapid change. The transition could be examined in more detail using boundary-later techniques if desired, but we confine our discussion in support of the conjectured narrow transition zone to examining the slope of $P(x)$ at its point of inflection $x_{*}\left(x_{*}>\mu_{1}\right)$, where $P^{\prime \prime}(x)$ changes sign. Setting $P^{\prime \prime}\left(x_{*}\right)=0$ in Eq. (42) we have

$$
\frac{P^{\prime}\left(x_{*}\right)^{2}}{P\left(x_{*}\right)^{2}}=2|\alpha| C\left[\frac{\beta}{|\alpha|}-P\left(x_{*}\right)\right] .
$$

However if we eliminate $P^{\prime}\left(x_{*}\right)$ using Eq. (43) we find a transcendental equation for $P\left(x_{*}\right)$ with no explicit dependence on $D$, although there is weak dependence on $D$ through $P\left(\mu_{1}\right)$ :

$$
\frac{\beta}{|\alpha|}+2 P\left(\mu_{1}\right)=3 P\left(x_{*}\right)+\frac{2 \beta}{|\alpha|} \log \left[\frac{P\left(\mu_{1}\right)}{P\left(x_{*}\right)}\right] .
$$

If we write $z_{*}=\lim _{D \rightarrow 0} P\left(x_{*}\right) / P\left(\mu_{1}\right)=(|\alpha| / \beta) \lim _{D \rightarrow 0} P\left(x_{*}\right)$ then we seek the solution $z_{*} \in(0,1)$ of $3 z-2 \log z=3$, and we find that $z_{*} \approx 0.417$. We deduce that as $D \rightarrow 0$,

$$
P^{\prime}\left(x_{*}\right) \sim-\left[\frac{2\left(1-z_{*}\right) \beta^{2} C}{|\alpha| D}\right]^{1 / 2} \approx-\frac{1.08 \beta}{|\alpha|}\left(\frac{\beta C}{D}\right)^{1 / 2},
$$

consistent with the hypothesis that in a small interval surrounding $x_{*}$, there is a very rapid transition between the solutions $P(x) \approx \beta /|\alpha|$ and $P(x) \approx 0$. For correct normalization of $P(x)$, the solutions $P(x) \approx \beta /|\alpha|$ must prevail for all but a small part of an interval of length $|\alpha| / \beta$.

We conclude (although we have not fully proved) that for $\alpha<0$,

$$
\lim _{D \rightarrow 0} P(x)= \begin{cases}\beta /|\alpha|, & \left|x-\mu_{1}\right|<|\alpha| /(2 \beta), \\ 0 & \left|x-\mu_{1}\right|>|\alpha| /(2 \beta),\end{cases}
$$

consistent with the only possible equilibrium solution of the diffusion-free equation (1) for this double-well potential. Our analysis has revealed how this limiting solution emerges asymptotically as the diffusivity is decreased and reveals how a small amount of added noise (numerical diffusion) could assist the deterministic system (1) to reach a mathematically discontinuous weak solution of the noiseless equilibrium problem, as found for the case $\beta=1, \alpha=1$ and $\mu_{1}=0$ by Fellner and Raoul [6].

\subsection{The potential $W(x)=\beta x^{2}+\delta x^{4}$}

Smooth double-well potentials (with $\delta>0$ and $\beta<0$ ) of this most basic polynomial type appear, for instance, in the modelling of cell aggregation [25, 26, 27, 28]. In the diffusion free aggregation model (1) the quartic-quadratic doublewell potential leads to the formation of precisely two aggregates, which converge in the large time behaviour to two Dirac masses. It is natural to ask how this behaviour is modified by adding diffusion. The scaling lengths contain the diffusion constant $D$, and it is of interest to return to the original variables and consider the small-diffusivity limit.

If we fix $\beta$ and $\delta$, with $\beta<0$, then the limit $D \rightarrow 0$ corresponds to $\theta \rightarrow-\infty$, and the asymptotic behaviour of $M$ given in Eq. (75) enables us to deduce that $\varpi$, defined in Eq. (64) as half of the distance between the two maxima in $P(x)$, converges to $[|\beta| /(8 \delta)]^{1 / 2}$. Moreover, if we hold $x, \beta$ and $\delta$ fixed (with $\beta<0$ ) and let $D \rightarrow 0$, we find that

$$
\frac{P(x)}{P\left(\mu_{1}\right)} \sim \exp \left\{-\frac{C}{D}\left[\delta\left(x-\mu_{1}\right)^{4}-\frac{|\beta|}{4}\left(x-\mu_{1}\right)^{2}\right]\right\},
$$

which can be written

$$
\frac{P(x)}{P\left(\mu_{1} \pm \varpi\right)} \sim \exp \left\{-\frac{C \delta}{D}\left[\left(x-\mu_{1}\right)^{2}-\varpi^{2}\right]^{2}\right\},
$$

showing that for sufficiently small $D$ the distribution condenses into two sharp spikes, whose separation $2 \varpi=[|\beta| /(2 \delta)]^{1 / 2}$ coincides with the distance at which the interaction force between two particles vanishes, consistent with the diffusion-free results and demonstrating how the presence of a small amount of noise enables the otherwise deterministic version of the problem to disaggregate the system mass into two equal tight clumps.

\section{Smoothed singular potentials and oscillatory solutions}

The ability of our model of cohesive stochastic swarms to recover in the $D \rightarrow 0$ limit results from hyperbolic aggregation models suggests that one might use our model to elucidate some puzzling aspects of the hyperbolic problem. It has been observed by Fellner and Raoul [6] that if the singular potential $W(x)=\alpha|x|+\beta x^{2}$ (with $\alpha<0$ ) is smoothed at the origin, then in the hyperbolic model the limiting distribution at long time is a finite string of approximately evenly spaced spikes, with the number of spikes related to the way in which the potential is smoothed. This phenomenon has so far proved difficult to explain.

Throughout this section, we shall always be considering the case $\alpha<0$ and where we wish to emphasise the signs of terms, we write $\alpha=-|\alpha|$, so that the singular potential of interest is written as $W(x)=-|\alpha||x|+\beta x^{2}$. We consider the smoothed potential

$$
W(x)= \begin{cases}\beta x^{2}-|\alpha||x|, & |x|>\epsilon, \\ \left(\beta-\frac{|\alpha|}{2 \epsilon}\right) x^{2}-\frac{|\alpha| \epsilon}{2}, & |x| \leq \epsilon,\end{cases}
$$


corresponding to

$$
W^{\prime}(x)= \begin{cases}2 \beta x-|\alpha| \operatorname{sgn}(x), & |x|>\epsilon, \\ 2 \beta x-\frac{|\alpha| x}{\epsilon}, & |x| \leq \epsilon,\end{cases}
$$

and

$$
W^{\prime \prime}(x)=2 \beta-\frac{|\alpha|}{\epsilon}[1-H(x-\epsilon)] H(x+\epsilon),
$$

where $H$ is the usual Heaviside step function. It follows from Eq. (40) that

$\frac{d^{2}}{d x^{2}} \log [P(x)]=\frac{P^{\prime \prime}(x)}{P(x)}-\frac{P^{\prime}(x)^{2}}{P(x)^{2}}=-\frac{2 \beta C}{D}+\frac{|\alpha| C}{\epsilon D} \int_{x-\epsilon}^{x+\epsilon} P(\xi) d \xi$.

\subsection{Observations for the finite mass case}

Let $x_{0}$ be a stationary point of $P(x)$, so that $P^{\prime}\left(x_{0}\right)=0$, and assume that $P\left(x_{0}\right)>0$. Then from Eq. (94) we have the inequality

$$
-\frac{2 \beta C}{D}<\frac{P^{\prime \prime}\left(x_{0}\right)}{P\left(x_{0}\right)}<-\frac{2 \beta C}{D}+\frac{|\alpha| C}{\epsilon D},
$$

where on the right-hand side we have replaced the integral over $\left(x_{0}-\epsilon, x_{0}+\epsilon\right)$ by the integral over $(-\infty, \infty)$. Since for $P(x)$ to have a local minimum at $x_{0}$ its second derivative must be nonnegative there, we can conclude rigorously that any equilibrium solution $P(x)$ with $D>0$ must be unimodal for $|\alpha|<2 \epsilon \beta$. (Although we have restricted our attention to $\alpha<0$, if we allow $\alpha$ to be of either sign, we conclude that the equilibrium distribution is unimodal at least for $\alpha>-2 \epsilon \beta$. It may be noted here that so long as $\alpha>-2 \epsilon \beta$, the potential $W(x)$ is cohesive at all distances, so the unimodality is not surprising in this case.)

We explore the possibility that for fixed $\alpha<0$ and fixed $\beta>0$, the equilibrium solution $P(x)$ might not be unimodal for some parameter values. Consider points $x_{\max }$ and $x_{\min }$, where $P(x)$ has, respectively, a local maximum (so $P^{\prime}\left(x_{\max }\right)=0$ and $\left.P^{\prime \prime}\left(x_{\max }\right) \leq 0\right)$ and a local minimum (so $P^{\prime}\left(x_{\min }\right)=0$ and $\left.P^{\prime \prime}\left(x_{\min }\right) \geq 0\right)$. It follows immediately from Eq. (94) that

$$
\frac{1}{2 \epsilon} \int_{x_{\max }-\epsilon}^{x_{\max }+\epsilon} P(\xi) d \xi \leq \frac{\beta}{|\alpha|}, \quad \frac{1}{2 \epsilon} \int_{x_{\min }-\epsilon}^{x_{\min }+\epsilon} P(\xi) d \xi \geq \frac{\beta}{|\alpha|} .
$$

Hence

$$
\min _{\left[x_{\max }-\epsilon, x_{\max }+\epsilon\right]} P \leq \frac{\beta}{|\alpha|}, \quad \max _{\left[x_{\min }-\epsilon, x_{\min }+\epsilon\right]} P \geq \frac{\beta}{|\alpha|} .
$$

These inequalities hold without any assumptions concerning the relative locations of the points $x_{\max }$ and $x_{\min }$. If we apply these inequalities to the case when $x_{\max }$ and $x_{\min }$ are adjacent extrema, they suggest that for adjacent maxima and minima,

$$
\left|x_{\max }-x_{\min }\right| \leq 2 \epsilon .
$$

This would be a rigorously derived conclusion if $P(x)$ were symmetric about both $x_{\max }$ and $x_{\min }$, which would imply periodic solutions $P(x)=P\left(x+2\left|x_{\max }-x_{\min }\right|\right)$ with infintely many peaks of equal height and thus infinite mass. However, in view of the compactly supported string of finitely many spikes produced by the diffusion free hyperbolic model for fixed small $\epsilon$, one expects (and observes numerically) that solutions of Eq. (94) for sufficiently small diffusivity $D$ feature a compactly supported string of finitely many smoothed spikes around the centre of mass with only slowly varying peak height. This oscillatory part of solutions of Eq. (94) for fixed $\epsilon$ and subsequently chosen small enough $D$ will in the following be approximated by periodic solutions of Eq. (94) with infinite mass. We believe that these periodic solutions are the simplest approach to understanding how the non-local term in Eq. (94) for any fixed $\epsilon>0$ and small enough diffusivity $D$ leads to oscillating solutions, while the limiting solutions for $\epsilon \rightarrow 0$ will always be unimodal.

We remark that we did not aim to demonstrate rigorously that such periodic, infinite mass solutions exist. Solutions of that type fall outside the framework of gradient flow solutions on probability spaces and the techniques that we have used on the infinite mass system in Sections 6.2-6.5 are based on other ideas. We note that is possible to make some progress on aggregation models producing periodic solutions by alternative means, if one is prepared to define the problem on a circle with restrictions on the potential related to the length circumference of the circle [48].

\subsection{A related infinite mass system}

We observe that Eq. (94) has the formal solution $P(x) \equiv$ $\beta /|\alpha|$ for all $x \in \mathbb{R}$. This is an infinite mass solution, so that $P(x)$ is no longer able to be interpreted as a probability density function. We have argued in Section 5.1 that for the finite-mass problem without smoothing, the solution is well approximated by $P(x) \approx \beta /|\alpha|$ for $|x|<|\alpha| / \beta$. Can an analysis of the infinite mass system shed any light on the low-diffusivity limit of the finite mass system?

Although $P(x) \equiv \beta /|\alpha|$ is a formal solution of an 'infinite mass' problem on $\mathbb{R}$ deduced from the $x$-derivative of the original governing equation under the assumption of equilibrium, we have noted in Section 2.1 that with the principal value interpretation (10) of all integrals involving $f(x-\xi)$, the problem has constant solutions.

For the remainder of Section 6 we address the question of the structure of non-constant periodic solutions. We have not been able to demonstrate rigorously that such solutions exist, but the analysis proceeds on the assumption that they do. The symmetry of the problem suggests that periodic solutions will exhibit reflection symmetry about any maxima or minima, so we restrict our discussion to even periodic solutions, of period $2 L$, say. Moreover, we shall assume that the periodic solution is unimodal in the sense that the only extrema within an interval of length $2 L$ centred on a maximum are minima at the endpoints of the interval. We are able to identify conditions that preclude such periodic solutions, conditions under which the existence of such periodic solutions becomes very plausible, and identify scaling features and qualitative properties of such periodic solutions in the small- $\epsilon$ limit. Reasons for restricting our attention to the cases $L<\epsilon$ and $\alpha \leq-2 \epsilon \beta$ will be explained shortly.

As the problem is translationally invariant, there is no loss of generality in considering even periodic solutions of period 
$2 L$, locating local maxima at $x=2 k L$ and local minima at $x=$ $(2 k+1) L$, where $k \in \mathbb{Z}$. This implies, in particular that

$$
P^{\prime \prime}(0) \leq 0 \leq P^{\prime \prime}(L)
$$

Exploiting the required reflection symmetry about each of $x=0$ and $x=L$ and noting that $P^{\prime}(0)=P^{\prime}(L)=0$, we deduce from Eq. (94) that

$$
\frac{P^{\prime \prime}(0)}{P(0)}-\frac{P^{\prime \prime}(L)}{P(L)}=\frac{2|\alpha| C}{\epsilon D}\left\{\int_{0}^{\epsilon} P(\xi) d \xi-\int_{L-\epsilon}^{L} P(\xi) d \xi\right\} .
$$

If $L>\epsilon$ we can exploit the monotonicity of $P$ on $(0, L)$ to deduce that the right-hand side of Eq. (100) is strictly positive, which contradicts the inequality (99). Hence equilibrium solutions with the expected symmetry properties must have period $2 L \leq 2 \epsilon$. If we seek to conserve mass relative to the uniform solution by insisting that

$$
\frac{1}{2 L} \int_{x-L}^{x+L} P(\xi) d \xi=\frac{\beta}{|\alpha|},
$$

then if $L=\epsilon$, we have $d^{2} / d x^{2} \log [P(x)] \equiv 0$, and non-constant periodic solutions are impossible. Our study of possible even periodic equilibrium solutions can therefore be restricted to solutions of period strictly less than $2 \epsilon$, that is, to the case $L<\epsilon$.

In Section 6.3 we conduct a time-dependent linear perturbation analysis to assess the stability of the equilibrium solution $P(x) \equiv \beta /|\alpha|$. Then in Section 6.4 we return to the harder, fully nonlinear problem of non-constant periodic solutions.

We note that in the ensuing analysis of the infinite mass system, no inequality constraint relating $\epsilon,|\alpha|$ and $\beta$ arises naturally. However we know that for the finite-mass system, the solution is unimodal if $\alpha>-2 \epsilon \beta$, and thus any interesting behaviour involving solutions for the infinite system with multiple local maxima can bring no insight to the finite mass system unless we have

$$
\alpha \leq-2 \epsilon \beta,
$$

a condition that we shall take from now on to have been prescribed.

\subsection{Linear stability analysis of equilibrium solutions}

We shall study the time-dependent response of the system to small perturbations away from an equilibrium solution $P(x)$ for $-\infty<x<\infty$ by writing

$$
p(x, t)=P(x)+\zeta \varphi(x, t), \quad \varphi(0,0)=1, \quad|\zeta| \ll 1 .
$$

Later in the analysis we shall restrict our attention to the case $P(x)=\beta /|\alpha|$ and the potential (91), but for the moment we keep the analysis more general. Retaining only $O(1)$ and $O(\zeta)$ terms in the drift-free $(v=0)$ case of the evolution equation $(20)$ we find that

$$
\begin{gathered}
\frac{\partial \varphi}{\partial t}=\frac{\partial}{\partial x}\left[D \frac{\partial \varphi}{\partial x}-C \varphi(x, t) \int_{-\infty}^{\infty} f(x-\xi) P(\xi) d \xi\right. \\
\left.-C P(x) \int_{-\infty}^{\infty} f(x-\xi) \varphi(\xi, y) d \xi\right] .
\end{gathered}
$$

Using the equilibrium equation (32) satisfied by $P(x)$ we can simplify this to

$$
\begin{aligned}
\frac{\partial \varphi}{\partial t}=\frac{\partial}{\partial x}\left[D \frac{\partial \varphi}{\partial x}\right. & -\frac{D P^{\prime}(x) \varphi(x, t)}{P(x)} \\
& \left.-C P(x) \int_{-\infty}^{\infty} f(x-\xi) \varphi(\xi, y) d \xi\right] .
\end{aligned}
$$

Selecting now $P(x)=\beta /|\alpha|$ and the potential (91) with associated force $f=-W^{\prime}$ [with $W^{\prime}$ given by Eq. (92)], and differentiating under the integral, we find that

$$
\frac{\partial \varphi}{\partial t}=D \frac{\partial^{2} \varphi}{\partial x^{2}}-\frac{C \beta}{\epsilon} \int_{x-\epsilon}^{x+\epsilon} \varphi(\xi, t) d \xi .
$$

Although the integral term is preceded by the factor $\beta$ associated with the quadratic component of the potential, it actually arises from the $-|\alpha||x|$ component of the potential (factors involving $|\alpha|$ cancel). To obtain Eq. (106), we have imposed two integral constraints, both of which are needed to remove the term

$$
\frac{d}{d x} \int_{-\infty}^{\infty} 2 \beta(x-\xi) \varphi(\xi, t) d \xi
$$

containing the effects of the quadratic component of the potential. The integral constraints are

$$
\int_{-\infty}^{\infty} \varphi(\xi, t) d \xi=0, \quad \int_{-\infty}^{\infty} \xi \varphi(\xi, t) d \xi \text { exists. }
$$

The first constraint ensures that the perturbation conserves mass. The second constraint holds provided that the perturbation function $\varphi(x, t)$ decays appropriately as $x \rightarrow \pm \infty$, but if we interpret the integral as a principal value and $\varphi(x, t)$ is symmetric about $x=0$, we do not need to be so restrictive about the large- $|x|$ behaviour. We now take as our fluctuation Ansatz a periodic perturbation of period $2 L=2 \pi / \lambda$ with a time-dependent amplitude:

$$
\varphi(x, t)=A(t) \cos (\lambda x), \quad A(0)=1 .
$$

This perturbation function transgresses the integral constraints (107) if they are interpreted in the classical sense, but they are satisfied if the integrals are interpreted in a principal value sense as the limit as $N \rightarrow \infty$ (with $N \in \mathbb{N}$ ) of integrals taken over the intervals $(-2 \pi N / \lambda, 2 \pi N / \lambda)$. Since we know that equilibrium periodic solutions must have period no greater than $2 \epsilon$, we hope to find at least some wavelengths $2 L \leq 2 \epsilon$ for which $A^{\prime}(t)>0$, leading to an instability of the uniform equilibrium solution, with evolution of the perturbed solution towards a non-constant periodic solution.

Using the integral

$$
\int_{x-\epsilon}^{x+\epsilon} \cos (\lambda \xi) d \xi=\frac{2 \sin (\lambda \epsilon)}{\lambda} \cos (\lambda x),
$$

it is easy to show the function defined by Eq. (108) is a solution of Eq. (106) if and only if $A(t)=e^{\kappa t}$, with

$$
\kappa=-2 C \beta\left[\Delta(\lambda \epsilon)^{2}+\frac{\sin (\lambda \epsilon)}{\lambda \epsilon}\right],
$$




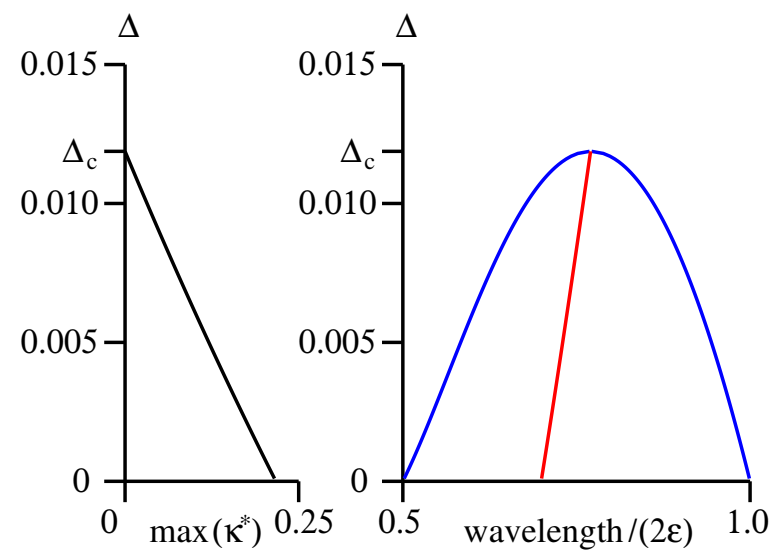

Figure 4: (Color online) In the plot on the right, the interval of wavelengths corresponding to unstable perturbations is shown in blue, while the red curve shows the wavelength with the largest growth rate $\kappa$ : this wavelength approaches a limit $\approx 0.69902$ as $\Delta \rightarrow 0$ and a limit $\approx 0.77036$ as $\Delta \rightarrow \Delta_{\mathrm{c}}$ from below. In the plot on the left, the dimensionless maximal growth rate $\kappa^{*}=\kappa /(2 C \beta)$ corresponding to a perturbation with the most unstable wavelength is shown (for the unstable $\Delta$ interval only).

where we have introduced the dimensionless diffusion constant

$$
\Delta=\frac{D}{2 C \beta \epsilon^{2}}
$$

It is important to notice that " small diffusion" requires the natural length scale $\sqrt{D /(2 C \beta)}$ associated with diffusion in the attractive potential to be small in comparison with the length scale associated with the smoothing of the repulsive part of the potential.

Can the perturbation grow with time? This requires the square-bracketed term in Eq. (110) to be negative. For the present, write $\theta=\lambda \epsilon$. We observe that $\sin \theta / \theta$ attains its global minimum on $[0, \infty)$ at $\theta_{\mathrm{c}} \approx 4.49341$, where it attains the value $-K(K \approx 0.217234)$, and $\sin \theta / \theta \geq 0$ for $0 \leq \theta \leq \pi$, while $\Delta \theta^{2}$ is monotonic increasing. We see at once that $\kappa<0$ for all $\theta>0$ (and so for all $\lambda>0$ ) if $\Delta \pi^{2}>K$. However, if $\Delta \theta_{\mathrm{c}}^{2}<K$, then there is at least one interval of $\lambda$ values for which $\kappa>0$.

It is now clear that there exists a critical dimensionless diffusivity $\Delta_{\mathrm{c}}$ such that the perturbations we have introduced decay to zero (stability) if $\Delta>\Delta_{\mathrm{c}}$, but perturbations of certain wavenumbers $\lambda$ (and corresponding wavelengths $2 L=2 \pi / \lambda$ ) have initial exponential growth with time when $\Delta<\Delta_{\mathrm{c}}$, suggesting that for $\Delta<\Delta_{\mathrm{c}}$ the system may evolve towards a nonuniform equilibrium state, and perhaps a periodic state.

Since $\sin \theta / \theta \geq 0$ for $0 \leq \theta \leq \pi$ it is clear that no perturbation with wavenumber $\lambda \leq \pi / \epsilon$ (wavelength $\geq 2 \epsilon$ ) is unstable, no matter how small $\Delta$ may be. A simple asymptotic analysis of the transcendental equation $\Delta \theta^{2}+\sin \theta / \theta=0$ shows that as $\Delta \rightarrow 0$, no perturbation of wavelength $\geq 2 \epsilon\left(1-\pi^{2} \Delta+\cdots\right)$ is unstable.

Using standard MATHEMATICA functions, we have determined numerically the critical dimensionless diffusivity

$$
\Delta_{\mathrm{c}} \approx 0.011876495
$$

and, for $\Delta<\Delta_{\mathrm{c}}$, the $\theta$-intervals and corresponding wavelength intervals that correspond to unstable perturbations and the wavelength corresponding to the largest positive value of the growth rate $\kappa$ : see Fig. 4.

Although we have studied only the initial growth of perturbations in the unstable case, and this analysis alone cannot give us strong information about possible periodic equilibrium solutions that arise in the long-time limit, it does encourage us to consider non-constant periodic solutions.

\subsection{Consistency of the delta function limit scenario}

From the preceding discussion we can restrict our study of periodic equilibrium solutions to the study of even periodic solutions of wavelength $2 L$, with $0<L<\epsilon$. We continue with notation introduced in Section 6.3, writing $\lambda=\pi / L$ and $\theta=\lambda \epsilon$, so that $\theta>\pi$. We shall write

$$
0 \leq P(x)=\sum_{n=0}^{\infty} P_{n} \cos (n \lambda x)=\exp \left[\sum_{n=0}^{\infty} Q_{n} \cos (n \lambda x)\right],
$$

and perform a number of formal manipulations without providing any rigorous proof of appropriate convergence. However, we note that any twice-differentiable solution of Eq. (94) is necessarily infinitely differentiable for all fixed $D>0$, so that the Fourier coefficients are rapidly decaying. We observe that from the orthogonality properties of the Fourier cosine functions,

$$
\int_{-L}^{L} P(x) d x=2 L P_{0}
$$

and so from Eq. (101), we have

$$
P_{0}=\frac{\beta}{|\alpha|}
$$

We now observe that Eqs (94) and (112) give

$$
\begin{aligned}
\frac{d^{2}}{d x^{2}} \sum_{n=0}^{\infty} Q_{n} \cos (n \lambda x) & =-\frac{2 \beta C}{D}+\frac{|\alpha| C}{\epsilon D} \int_{x-\epsilon}^{x+\epsilon} \sum_{n=0}^{\infty} P_{n} \cos (n \lambda \xi) d \xi \\
& =\frac{|\alpha| C}{\epsilon D} \sum_{n=1}^{\infty} P_{n} \int_{x-\epsilon}^{x+\epsilon} \cos (n \lambda \xi) d \xi
\end{aligned}
$$

If we exploit the integral (109) with $\lambda$ replaced by $n \lambda$ we find without difficulty that

$$
Q_{n}=-\frac{2|\alpha| C \sin (n \lambda \epsilon)}{\epsilon D(n \lambda)^{3}} P_{n}, \quad n \geq 1 .
$$

If we now rescale the Fourier coefficients by writing

$$
P_{n}=\frac{\beta}{|\alpha|} R_{n} \quad(n \geq 1), \quad \exp \left(Q_{0}\right)=\frac{\beta}{|\alpha|} R_{0},
$$

and we write $\Delta=D /\left(2 \beta C \epsilon^{2}\right)$ as before, we find that

$$
1+\sum_{n=1}^{\infty} R_{n} \cos (n \lambda x)=R_{0} \exp \left[-\sum_{n=1}^{\infty} \frac{R_{n} \sin (n \lambda \epsilon) \cos (n \lambda x)}{\Delta(n \lambda \epsilon)^{3}}\right] .
$$


As we now show, for any $\Delta>0$ there cannot be a non-constant periodic solution with period precisely $2 \epsilon$. To see this note that if $L=\epsilon$, then $\lambda=\pi / \epsilon$ and $\operatorname{sos} \sin (n \lambda \epsilon)=0$ for all $n \geq 1$, leading to

$$
1+\sum_{n=1}^{\infty} R_{n} \cos (n \lambda x)=R_{0}
$$

Then from the orthogonality of the cosines, $R_{0}=1$ and $R_{n}=0$ for all $n \geq 1$.

Considering the nonlinear and nonlocal problem (94), a rigorous resolution of the question of the existence, at least for sufficiently small but nonzero $\Delta$, of periodic solutions with period $2 L$ not precisely equal to $2 \epsilon$ appears difficult. We can, however, explore the consistency of the hypothesis (inspired by work on the diffusion-free problem $[5,6])$ that as $\Delta \rightarrow 0$, we obtain a set of periodically spaced delta functions for $P(x)$. If we recall the generalized function identity [49]

$$
\sum_{n=-\infty}^{\infty} \delta(x-2 n L)=\frac{1}{2 L}+\sum_{n=1}^{\infty} \frac{\cos (n \pi x / L)}{L},
$$

then for the $D \rightarrow 0$ limit to produce a string of delta functions with $L=\pi / \lambda$, consistent with the constraint (114), we need to obtain as our limiting form of $P(x)$,

$$
\frac{2 \pi \beta}{\lambda|\alpha|} \sum_{n=-\infty}^{\infty} \delta(x-2 n \pi / \lambda)=\frac{\beta}{|\alpha|}+\frac{2 \beta}{|\alpha|} \sum_{n=1}^{\infty} \cos (n \lambda x),
$$

corresponding to $R_{n} \rightarrow 2$ as $\Delta \rightarrow 0$.

From Eq. (118), writing $\lambda x=\vartheta$ for brevity, we have from the usual formula for the Fourier coefficients that

$$
R_{m}=\frac{2 R_{0}}{\pi} \int_{0}^{\pi} \cos (m \vartheta) \exp \left[-\sum_{n=1}^{\infty} \frac{R_{n} \sin (n \lambda \epsilon) \cos (n \vartheta)}{\Delta(n \lambda \epsilon)^{3}}\right] d \vartheta
$$

We shall write $\theta_{\Delta}=\lambda \epsilon$. As in the linear stability analysis the longest-wavelength unstable perturbations occur with $\theta$ in a proper subinterval of $(\pi, 2 \pi)$ which expands to fill the interval as $\Delta \rightarrow 0$ (see Fig. 4), we shall assume that $\theta_{\Delta} \in(\pi, 2 \pi)$, but we have to leave open the possibility that either $\theta_{\Delta} \rightarrow \pi$ or $\theta_{\Delta} \rightarrow 2 \pi$ as $\Delta \rightarrow 0$.

$$
\begin{aligned}
& \text { If } R_{n} \rightarrow 2 \text { for all } n \geq 1 \text { as } \Delta \rightarrow 0, \text { we must have } \\
& R_{m} \sim \frac{2 R_{0}}{\pi} \int_{0}^{\pi} \cos (m \vartheta) \exp \left[-\sum_{n=1}^{\infty} \frac{2 \sin \left(n \theta_{\Delta}\right) \cos (n \vartheta)}{\Delta\left(n \theta_{\Delta}\right)^{3}}\right] d \vartheta
\end{aligned}
$$

Our task now is to verify that the dominant asymptotic form of the right-hand side is 2 , to lend support for the hypothesis that $R_{n} \rightarrow 2$. The argument of the exponential is a continuously differentiable function of $\vartheta$, and so its dominant asymptotic form can be extracted using Laplace's method [50].

We observe that

$$
\begin{aligned}
\sum_{n=1}^{\infty} \frac{2 \sin \left(n \theta_{\Delta}\right) \cos (n \vartheta)}{\Delta\left(n \theta_{\Delta}\right)^{3}}=\frac{1}{\Delta \theta_{\Delta}^{3}}\left\{\sum_{n=1}^{\infty} \frac{\sin \left[n\left(\theta_{\Delta}+\vartheta\right)\right]}{n^{3}}\right. \\
\left.+\sum_{n=1}^{\infty} \frac{\sin \left[n\left(\theta_{\Delta}-\vartheta\right)\right]}{n^{3}}\right\}
\end{aligned}
$$

From known identities for Bernoulli polynomials [47] we have the identity

$$
\sum_{n=1}^{\infty} \frac{\sin (n z)}{n^{3}}=\frac{1}{12}\left[z^{3}-3 \pi z^{2} \operatorname{sgn}(z)+2 \pi^{2} z\right], \quad-2 \pi \leq z \leq 2 \pi .
$$

Given that $\pi<\theta_{\Delta}<2 \pi$, and that in the integration we have $0<\vartheta<\pi$, we see that $0<\theta_{\Delta}-\vartheta<2 \pi$. Hence we can use the identity (124) with $z=\theta_{\Delta}-\vartheta$ and in this case $\operatorname{sgn}(z)=1$. However, we cannot use the identity (124) directly with $z=$ $\theta_{\Delta} \pm \vartheta$ everywhere in the integral. Although we always have $\theta_{\Delta}+\vartheta>0$, we only have $\theta_{\Delta}+\vartheta<2 \pi$ for part of the integration interval. If $\theta_{\Delta}+\vartheta>2 \pi$ we note that

$$
\sin \left[n\left(\theta_{\Delta}+\vartheta\right)\right]=\sin \left[n\left(\theta_{\Delta}+\vartheta-2 \pi\right)\right] .
$$

and we shall use the identity (124) with $z=\theta_{\Delta}+\vartheta-2 \pi$. It is easy to verify that in this case we have $0<z<2 \pi$. We now have

$$
\begin{aligned}
R_{m} \sim & \frac{2 R_{0}}{\pi} \int_{0}^{2 \pi-\theta_{\Delta}} \cos (m \vartheta) \exp \left[-\frac{Q_{1}(\vartheta)}{12 \Delta \theta_{\Delta}^{3}}\right] d \vartheta \\
& +\frac{2 R_{0}}{\pi} \int_{2 \pi-\theta_{\Delta}}^{\pi} \cos (m \vartheta) \exp \left[-\frac{Q_{2}(\vartheta)}{12 \Delta \theta_{\Delta}^{3}}\right] d \vartheta
\end{aligned}
$$

where $Q_{1}(\vartheta)$ and $Q_{2}(\vartheta)$ are quadratic polynomial functions, which can be written tidily as

$$
\begin{aligned}
& Q_{1}(\vartheta)=-2 \theta_{\Delta}\left(\theta_{\Delta}-\pi\right)\left(2 \pi-\theta_{\Delta}\right)+6\left(\theta_{\Delta}-\pi\right) \vartheta^{2}, \\
& Q_{2}(\vartheta)=-6\left(2 \pi-\theta_{\Delta}\right)(\pi-\vartheta)^{2}+2\left(\theta_{\Delta}-\pi\right)\left(2 \pi-\theta_{\Delta}\right)\left(3 \pi-\theta_{\Delta}\right) .
\end{aligned}
$$

Once we locate their minima within the corresponding integration intervals, we can estimate the asymptotic behaviour of the integral using Laplace's method [50].

The function $Q_{1}(\vartheta)$ takes its minimum on $\left[0,2 \pi-\theta_{\Delta}\right]$ at $\vartheta=0$ and so from Laplace's method we deduce at once that as $\Delta \rightarrow 0$,

$$
\begin{aligned}
\int_{0}^{2 \pi-\theta_{\Delta}} & \cos (m \vartheta) \exp \left[-\frac{Q_{1}(\vartheta)}{12 \Delta \theta_{\Delta}^{3}}\right] d \vartheta \\
& \sim \exp \left[-\frac{Q_{1}(0)}{12 \Delta \theta_{\Delta}^{3}}\right] \int_{0}^{\infty} \exp \left[-\frac{\left(\theta_{\Delta}-\pi\right) \vartheta^{2}}{2 \Delta \theta_{\Delta}^{3}}\right] d \vartheta \\
= & \frac{1}{2}\left(\frac{2 \pi \Delta \theta_{\Delta}^{3}}{\theta_{\Delta}-\pi}\right)^{1 / 2} \exp \left[\frac{\left.\left(\theta_{\Delta}-\pi\right)\left(2 \pi-\theta_{\Delta}\right)\right)}{6 \Delta \theta_{\Delta}^{2}}\right]
\end{aligned}
$$

We note that the leading order behaviour as $\Delta \rightarrow 0$ is independent of $m$.

The function $Q_{2}(\vartheta)$ attains its minimum value on $\left[2 \pi-\theta_{\Delta}, \pi\right]$ at $\vartheta=2 \pi-\theta_{\Delta}$ and so

$$
\min _{\vartheta \in\left[2 \pi-\theta_{\Delta}, \pi\right]} Q_{2}(\vartheta)>\min _{\vartheta \in\left[0,2 \pi-\theta_{\Delta}\right]} Q_{1}(\vartheta) .
$$

It follows that the second integral in Eq. (125) is exponentially small compared to the first integral, and thus if we assume the 
hypotheses that as $\Delta \rightarrow 0$ we have $R_{n} \rightarrow 2$ for $n \geq 1$ while $\theta_{\Delta} \in(\pi, 2 \pi)$, we predict that for $m \geq 1$,

$$
R_{m} \sim \frac{R_{0}}{\pi}\left(\frac{2 \pi \Delta \theta_{\Delta}^{3}}{\theta_{\Delta}-\pi}\right)^{1 / 2} \exp \left[\frac{\left.\left(\theta_{\Delta}-\pi\right)\left(2 \pi-\theta_{\Delta}\right)\right)}{6 \Delta \theta_{\Delta}^{2}}\right] .
$$

We now see that for this to be consistent with $R_{m} \rightarrow 2$ for all $m \geq 1$ we only need $R_{0}$ to behave appropriately:

$$
R_{0} \sim 2 \pi\left(\frac{\theta_{\Delta}-\pi}{2 \pi \Delta \theta_{\Delta}^{3}}\right)^{1 / 2} \exp \left[-\frac{\left.\left(\theta_{\Delta}-\pi\right)\left(2 \pi-\theta_{\Delta}\right)\right)}{6 \Delta \theta_{\Delta}^{2}}\right] .
$$

This important condition will be revisited in Section 6.5.

\subsection{An asymptotic analysis}

The rigorous and heuristic conclusions in Sections 6.2-6.4 motivate us to attempt to construct periodic solutions of the equilibrium version of Eq. (94) that consist of narrow peaks that contain most of the mass and valleys containing very little mass. We shall write

$$
x=\epsilon X \quad \text { and } \quad P(x)=\frac{\beta}{|\alpha|} R(x / \epsilon)=\frac{\beta}{|\alpha|} R(X)
$$

(scaling space against the smoothing distance $\epsilon$ ) and introduce $\Delta=D /\left(2 \beta C \epsilon^{2}\right)$ as before, so that we need to solve

$$
\frac{d^{2}}{d X^{2}} \log [R(X)]=-\frac{1}{\Delta}+\frac{1}{2 \Delta} \int_{X-1}^{X+1} R(\zeta) d \zeta .
$$

We note that $R(X) \equiv 1$ is a solution, but now we seek (in the limit $\Delta \rightarrow 0$ ) non-constant solutions $R_{\Delta}(X)$ of (minimal) period $\tau_{\Delta}<2$ (corresponding to $L<\epsilon$ ). We note that from periodicity

$$
\int_{X}^{X+\tau_{\Delta}} R_{\Delta}(X) d X=A_{\Delta}=\text { constant for all } X,
$$

so that for all $n \in \mathbb{N}$,

$$
\int_{0}^{n \tau_{\Delta}} R_{\Delta}(X) d X=n A_{\Delta}
$$

To conserve mass compared to the uniform solution $R(X) \equiv 1$, we shall require that

$$
\int_{0}^{\ell} R_{\Delta}(X) d X \sim \ell \quad \text { as } \ell \rightarrow \infty .
$$

and on setting $\ell=n \tau_{\Delta}$ and letting $n \rightarrow \infty$, we conclude from Eqs (134) and (135) that $A_{\delta}=\tau_{\delta}$. It follows that for nonconstant periodic solutions the maximum value attained over a period is strictly greater than 1 , and the minimum value is strictly less than 1 .

In the linear stability analysis, we predicted instability (suggesting evolution towards an equilibrium non-constant periodic solution) once $\Delta$ is sufficiently small, with the largest wavelength unstable perturbations corresponding to a subinterval of the interval $\pi<\lambda \epsilon<2 \pi$. Under the scalings that we are now using, $\epsilon \tau_{\Delta}=2 \pi / \lambda$ and so we anticipate that $1<\tau_{\Delta}<2$. The right-hand side of Eq. (132) vanishes identically if $2=k \tau_{\Delta}$ for any $k \in \mathbb{N}$, so non-constant solutions with periods $\tau_{\Delta}=2 / k$ are excluded.

In the careful and rather detailed analysis that follows we make the following assumptions as working hypotheses.
1. In an interval $0<\Delta<\Delta_{1}$ the integrodifferential equation (132) has a non-constant periodic solution $R_{\Delta}(X)$ (with period $\tau_{\Delta}$ ) that is symmetric under reflection about any local maximum and or minimum and has precisely one local maximum and one local minimum associated with each interval of length $\tau_{\Delta}$.

Given this first hypothesis (largely a restatement of assumptions that we explained in Section 6.2 would be made throughout Section 6) and the translational invariance of the problem, there is no loss of generality in locating the maxima at $X=m \tau_{\Delta}$ and the minima at $(m+1 / 2) \tau_{\Delta}$, where $m \in \mathbb{Z}$.

2. The function $R_{\Delta}(X)$ is non-negative and

$$
\int_{0}^{\tau_{\Delta}} R_{\Delta}(X) d X=2 \int_{0}^{\tau_{\Delta} / 2} R_{\Delta}(X) d X=\tau_{\Delta} .
$$

3. For $0<\Delta<\Delta_{1}$, we have $\tau_{\Delta} \in(1,2)$ and

$$
\tau_{0}=\lim _{\Delta \rightarrow 0^{+}} \tau_{\Delta} \in[1,2] \text { exists. }
$$

4. We do not have $\lim _{\Delta \rightarrow 0} R_{\Delta}(X) \equiv 1$.

Concerning the last of these hypotheses we note that as $\Delta$ decreases, the growth rate for fastest-growing perturbation in the linear stability analysis increases. If there are non-constant periodic equilibrium solutions for some nonzero value of $\Delta$, we would expect these to be retained and to maintain or increase the amplitude of their spatial oscillations as $\Delta$ is decreased.

\subsubsection{Properties of non-constant periodic solutions $R_{\Delta}(X)$}

Because of the necessity of a concavity change in the periodic function $\log \left(R_{\Delta}\right)$ we see from Eq. (132) that

$$
\mu_{\Delta}=\min _{X} \int_{X-1}^{X+1} R_{\Delta}(\zeta) d \zeta \leq 2, \quad v_{\Delta}=\max _{X} \int_{X-1}^{X+1} R_{\Delta}(\zeta) d \zeta \geq 2
$$

and so

$$
-\frac{\left(2-\mu_{\Delta}\right)}{2 \Delta} \leq \frac{d^{2}}{d X^{2}} \log \left[R_{\Delta}(X)\right] \leq \frac{\left(v_{\Delta}-2\right)}{2 \Delta} .
$$

Noting that the first derivative of $\log \left[R_{\Delta}(X)\right]$ vanishes at the extremal point $X=0$, we find from integrating the inequality that

$$
-\frac{\left(2-\mu_{\Delta}\right)}{4 \Delta} X^{2} \leq \log \left[\frac{R_{\Delta}(X)}{R_{\Delta}(0)}\right] \leq \frac{\left(v_{\Delta}-2\right)}{4 \Delta} X^{2} .
$$

For $\Delta<\min \left(1 / 4, \Delta_{1}\right)$ we have $0<\Delta^{1 / 2}<\tau_{\Delta} / 2$ and we see from Eq. (139) that

$$
R_{\Delta}(0) e^{-\left(2-\mu_{\Delta}\right) / 4} \leq R_{\Delta}\left(\Delta^{1 / 2}\right) \leq R_{\Delta}(0) e^{\left(v_{\Delta}-2\right) / 4} .
$$

Since $R_{\Delta}(X)$ decreases on $\left[0, \tau_{\Delta} / 2\right]$ we see that

$$
\begin{aligned}
\Delta^{1 / 2} R_{\Delta}(0) & \geq \int_{0}^{\Delta^{1 / 2}} R_{\Delta}(X) d X \\
& \geq \Delta^{1 / 2} R_{\Delta}\left(\Delta^{1 / 2}\right) \geq \Delta^{1 / 2} R_{\Delta}(0) e^{-\left(2-\mu_{\Delta}\right) / 4} .
\end{aligned}
$$


Also, recalling Eq. (136),

$$
\int_{0}^{\Delta^{1 / 2}} R_{\Delta}(X) d X<\int_{0}^{\tau_{\Delta} / 2} R_{\Delta}(X) d X=\frac{\tau_{\Delta}}{2},
$$

so (noting that $\tau_{\Delta}<2$ and $\mu_{\Delta}>0$ ) we have the upper bound

$$
R_{\Delta}(0)<\frac{\tau_{\Delta}}{2 \Delta^{1 / 2}} e^{\left(2-\mu_{\Delta}\right) / 4}<\frac{e^{1 / 2}}{\Delta^{1 / 2}} .
$$

Thus, although $R_{\Delta}(0)$ may grow unboundedly as $\Delta \rightarrow 0$, its growth rate is constrained.

Next, we observe that

$$
\begin{aligned}
\int_{X-1}^{X+1} R_{\Delta}(\zeta) d \zeta= & \int_{-1}^{1} R_{\Delta}(X+Y) d Y \\
= & \int_{-1}^{-\tau_{\Delta} / 2} R_{\Delta}(X+Y) d Y+\int_{-\tau_{\Delta} / 2}^{\tau_{\Delta} / 2} R_{\Delta}(X+Y) d Y \\
& +\int_{\tau_{\Delta} / 2}^{1} R_{\Delta}(X+Y) d Y
\end{aligned}
$$

Exploiting periodicity we can add $\tau_{\Delta}$ to both terminals in the first integral on the right, while we know that the second integral is $\tau_{\Delta}$. Hence

$$
\int_{X-1}^{X+1} R_{\Delta}(\zeta) d \zeta=\tau_{\Delta}+\int_{\tau_{\Delta}-1}^{1} R_{\Delta}(X+Y) d Y
$$

We now define a new integration variable $Z=Y-\tau_{\Delta} / 2$ and we reformulate the integrodifferential equation (132) as

$$
\frac{d^{2}}{d X^{2}} \log \left[R_{\Delta}(X)\right]=-\frac{\delta_{\Delta}}{\Delta}+\frac{1}{2 \Delta} \int_{-\delta_{\Delta}}^{\delta_{\Delta}} R_{\Delta}\left(\frac{\tau_{\Delta}}{2}+X+Z\right) d Z
$$

where

$$
0<\delta_{\Delta} \stackrel{\text { def }}{=} 1-\frac{\tau_{\Delta}}{2}<\frac{1}{2} .
$$

The last inequality arises from $\tau_{\Delta} \in(1,2)$. We remark that the reduction of the length of the integration interval from 2 to $2 \delta_{\Delta}=2-\tau_{\Delta}$ turns out to be of significant practical importance in the ensuing analysis.

We introduce the rescaled function $Q_{\Delta}(X)=\Delta \log \left[R_{\Delta}(X)\right]$, so that

$$
R_{\Delta}(X)=\exp \left[\Delta^{-1} Q_{\Delta}(X)\right]
$$

and

$$
Q_{\Delta}^{\prime \prime}(X)=-\delta_{\Delta}+\frac{1}{2} \int_{-\delta_{\Delta}}^{\delta_{\Delta}} R_{\Delta}\left(\frac{\tau_{\Delta}}{2}+X+Z\right) d Z .
$$

Setting $X=0$ and $X=\tau_{\Delta} / 2$ (exploiting periodicity in the latter case) we have from Eq. (149) that

$$
\begin{aligned}
Q_{\Delta}^{\prime \prime}(0) & =-\delta_{\Delta}+\frac{1}{2} \int_{-\delta_{\Delta}}^{\delta_{\Delta}} R\left(\frac{\tau_{\Delta}}{2}+Z\right) d Z \leq 0, \\
Q_{\Delta}^{\prime \prime}\left(\tau_{\Delta} / 2\right) & =-\delta_{\Delta}+\frac{1}{2} \int_{-\delta_{\Delta}}^{\delta_{\Delta}} R_{\Delta}(Z) d Z \geq 0 .
\end{aligned}
$$

The inequalities reflect concavity constraints that follow from the status of 0 and $\tau_{\Delta} / 2$ as maxima and minima, respectively.
Since the integral in Eq. (149) is no greater than $\tau_{\Delta}$ we are able to conclude that

$$
-\delta_{\Delta}<Q_{\Delta}^{\prime \prime}(X)<1-2 \delta_{\Delta}
$$

and so we have uniform second-derivative bounds for our rescaled function $Q_{\Delta}(X)$ as $\Delta \rightarrow 0$, namely $-1 / 2<Q_{\Delta}^{\prime \prime}(X)<1$.

We can now extract some useful information on the behaviour of $\delta_{\Delta}$ as $\Delta \rightarrow 0$. From the second-order Mean Value Theorem, and the status of 0 and $\tau_{\Delta} / 2$ as maxima and minima, respectively, of $R_{\Delta}$ (and of $Q_{\Delta}$ ) we have on the interval $0 \leq X \leq \tau_{\Delta}$

$$
Q_{\Delta}(X) \leq Q_{\Delta}\left(\tau_{\Delta} / 2\right)+\frac{\delta_{\Delta}}{2}\left[R_{\Delta}(0)-1\right]\left(X-\frac{\tau_{\Delta}}{2}\right)^{2} .
$$

Since from the discussion following Eq. (135) the integral over one period is $\tau_{\Delta}$, we have $R\left(\tau_{\Delta} / 2\right) \leq 1$, while we know from the inequality (143) that $R_{\Delta}(0) \leq(e / \Delta)^{1 / 2}$. We arrive at the upper bound

$$
R_{\Delta}(X) \leq \exp \left\{\frac{\delta_{\Delta}}{2 \Delta}\left[\left(\frac{e}{\Delta}\right)^{1 / 2}-1\right] \frac{\tau_{\Delta}^{2}}{4}\right\} \quad \text { for } \quad 0 \leq X \leq \tau_{\Delta} .
$$

If $\delta_{\Delta}=o\left(\Delta^{3 / 2}\right)$ we have $\lim \sup _{\Delta \rightarrow 0} R_{\Delta}(X) \leq 1$ (uniformly in $X)$. Given the monotonicity of $R$ on $\left(0, \tau_{\Delta} / 2\right)$ and $\left(\tau_{\Delta} / 2, \tau_{\Delta}\right)$ and the requirement that the integral over a period is $\tau_{\Delta}$, this leads to $\lim _{\Delta \rightarrow 0} R_{\Delta}(X) \equiv 1$, violating our fourth working hypothesis. Thus in what follows, although we may have $\delta_{\Delta} \rightarrow 0$ as $\Delta \rightarrow 0$ (corresponding to $\tau_{\Delta} \rightarrow 2$ ), we must assume that

$$
\frac{\delta_{\Delta}}{\Delta^{3 / 2}} \nrightarrow 0
$$

From the second-order mean value theorem, using the inequality (152) we have the bounds

$Q(X) \geq Q(0)-\frac{\delta_{\Delta} X^{2}}{2}, \quad Q(X) \leq Q\left(\frac{\tau_{\Delta}}{2}\right)+\frac{\left(1-2 \delta_{\Delta}\right)}{2}\left(X-\frac{\tau_{\Delta}}{2}\right)^{2}$.

Using Eq. (148) and integrating the resulting inequalities for $R(X)$ over the intervals of length $\tau_{\Delta}$ centred on 0 and $\tau_{\Delta} / 2$ respectively, we find that

$$
\begin{aligned}
& \tau_{\Delta} \geq R_{\Delta}(0) \int_{-\tau_{\Delta} / 2}^{\tau_{\Delta} / 2} \exp \left(-\frac{\delta_{\Delta} X^{2}}{2 \Delta}\right) d X \\
& \tau_{\Delta} \leq R_{\Delta}\left(\frac{\tau_{\Delta}}{2}\right) \int_{-\tau_{\Delta} / 2}^{\tau_{\Delta} / 2} \exp \left(\frac{\left(1-2 \delta_{\Delta}\right) X^{2}}{2 \Delta}\right) d X .
\end{aligned}
$$

Since $R_{\Delta}(\tau / 2) \leq R_{\Delta}(X) \leq R_{\Delta}(0)$ and the integral of $R_{\Delta}(X)$ over a period is $\tau_{\Delta}$, if either of the cases

$$
\lim _{\Delta \rightarrow 0} R_{\Delta}(0)=1 \quad \text { or } \quad \lim _{\Delta \rightarrow 0} R_{\Delta}\left(\frac{\tau_{\Delta}}{2}\right)=1
$$

arises, then $R_{\Delta}(X) \rightarrow 1$ (in the $L^{1}$ sense, at least) as $\Delta \rightarrow 0$ and we lose the non-constant periodic solution, violating our working hypotheses. Therefore we need to prevent the integrands in each of the inequalities (156) and (157) from converging to 1 as $\Delta \rightarrow 0$. Thus we conclude that as $\Delta \rightarrow 0$,

$$
\frac{\delta_{\Delta}}{\Delta} \nrightarrow 0 \text { and } \quad \frac{\left(1-2 \delta_{\Delta}\right)}{\Delta} \nrightarrow 0 .
$$


In terms if the limiting behaviour of the period $\tau_{\Delta}$, we see that neither $\tau_{\Delta}=1+o(\Delta)$ nor $\tau_{\Delta}=2-o(\Delta)$ is compatible with the working hypotheses.

There are additional important conclusions that can be drawn from the inequality (156), given that we have rejected the case $\delta_{\Delta} / \Delta \rightarrow 0$.

(i) If $\delta_{\Delta} / \Delta$ is bounded above as $\Delta \rightarrow 0$, then $R_{\Delta}(0)$ is also bounded above. This prevents the periodic solution $R_{\Delta}(X)$ converging to a string of delta functions.

(ii) If $\delta_{\Delta} / \Delta \rightarrow \infty$ as $\Delta \rightarrow 0$, then we have

$$
R_{\Delta}(0)\left[\left(\frac{2 \pi \Delta}{\delta_{\Delta}}\right)^{1 / 2}+o(1)\right] \leq \tau_{\Delta}
$$

If $\delta_{\Delta} \rightarrow 0$ as $\Delta \rightarrow 0$, this is a sharper result than the bound (143).

\subsubsection{Asymptotics of non-constant periodic solutions $R_{\Delta}(X)$}

Up to the present stage of our analysis, we have made a minimal number of conjectures about exact properties of the nonconstant periodic solution, but we have not made any mathematical approximations or any assumptions concerning asymptotic behaviour. We shall now commence our analysis, with progressively increasing refinement, of the conjectured nonconstant periodic solution.

We begin with a first approximation of the solution by replacing the solution in the vicinity of its maximum at $X=0$ by a Gaussian:

$$
R_{\Delta}(X) \approx R_{\Delta}(0) \exp \left[-\frac{\left|Q_{\Delta}^{\prime \prime}(0)\right| X^{2}}{2 \Delta}\right]
$$

From the second-order Mean Value Theorem, this approximation asserts that $Q_{\Delta}^{\prime \prime}(X)$ is almost constant near $X=0$. If $\Delta \ll$ 1 , there is an exponentially small contribution to the integral outside of an interval of width $O\left(\left[\Delta / Q_{\Delta}^{\prime \prime}(0) \mid\right]^{1 / 2-a}\right)$ centred on the origin ( $a>0$ fixed but arbitrary) and so integrating over $-\tau_{\Delta} / 2<X<\tau_{\Delta} / 2$ and then extending the terminals on the integral of the Gaussian to infinity, we deduce that the mass associated with the Gaussian peak is

$$
m \approx R_{\Delta}(0)\left[\frac{2 \Delta \pi}{\left|Q_{\Delta}^{\prime \prime}(0)\right|}\right]^{1 / 2} .
$$

If we now replace $X$ by $X+\tau_{\Delta} / 2$ in Eq. (149), use periodicity of $R$ and insert the Gaussian approximation (160) we see that

$$
\begin{aligned}
Q_{\Delta}^{\prime \prime}\left(\tau_{\Delta} / 2+X\right) & \approx-\delta_{\Delta}+\frac{R_{\Delta}(0)}{2} \int_{-\delta_{\Delta}}^{\delta_{\Delta}} \exp \left[-\frac{\left|Q_{\Delta}^{\prime \prime}(0)\right|(X+Z)^{2}}{2 \Delta}\right] d Z \\
& =-\delta_{\Delta}+\frac{R_{\Delta}(0)}{2} \int_{X-\delta_{\Delta}}^{X+\delta_{\Delta}} \exp \left[-\frac{\left|Q_{\Delta}^{\prime \prime}(0)\right| Z^{2}}{2 \Delta}\right] d Z
\end{aligned}
$$

For small $\Delta$ the integral with be exponentially small if $X+\delta_{\Delta}<$ 0 or if $X-\delta_{\Delta}>0$, since in these cases the peak of the Gaussian lies outside the integration interval. On the other hand,when
$-\delta_{\Delta}<X<\delta_{\Delta}$ we can extend the integration interval to $(-\infty, \infty)$ incurring an exponentially small error and we find that

$$
Q_{\Delta}^{\prime \prime}\left(\tau_{\Delta} / 2+X\right) \approx-\delta_{\Delta}+\frac{R_{\Delta}(0)}{2}\left[\frac{2 \Delta \pi}{\left|Q_{\Delta}^{\prime \prime}(0)\right|}\right]^{1 / 2} .
$$

Eliminating $R_{\Delta}(0)$ from Eqs (161) and (163) we now find that

$$
Q_{\Delta}^{\prime \prime}\left(\tau_{\Delta} / 2+X\right) \approx-\delta_{\Delta}+\frac{m}{2}=\frac{m+\tau_{\Delta}}{2}-1 \text { if }-\delta_{\Delta}<X<\delta_{\Delta} .
$$

Thus at our present level of approximate analysis, where $n \in \mathbb{Z}$,

$Q_{\Delta}^{\prime \prime}(X)= \begin{cases}\frac{m+\tau_{\Delta}}{2}-1, & \left(n+\frac{1}{2}\right) \tau_{\Delta}-\delta_{\Delta}<X<\left(n+\frac{1}{2}\right) \tau_{\Delta}+\delta_{\Delta}, \\ -\delta_{\Delta}, & \text { otherwise. }\end{cases}$

Of course at small but nonzero $\Delta$, rather than having a discontinuous switch between the two allowed values, there will be a very narrow transition region, in which the leading-order behaviour can be represented in terms of the error function $\operatorname{erf}(\cdot)$, using Eq. (162). The result that $Q_{\Delta}^{\prime \prime}(X) \approx-\delta_{\Delta}$ except close to the minima of $R$ is consistent with Eq. (150) if the neighbourhood of a minimum in $R$ subtends negligible area.

If we integrate Eq. (165) from $\tau_{\Delta} / 2$ to $X \in\left[\tau_{\Delta} / 2, \tau_{\Delta}\right]$ and recall that $Q_{\Delta}^{\prime}\left(\tau_{\Delta} / 2\right)=0$, we find that

$Q_{\Delta}^{\prime}\left(X+\frac{\tau_{\Delta}}{2}\right) \approx \begin{cases}\left(\frac{m+\tau_{\Delta}}{2}-1\right) X, & 0 \leq X<\delta_{\Delta}, \\ \left(\frac{m+\tau_{\Delta}}{2}-1\right) \delta_{\Delta}-\delta_{\Delta}\left(X-\delta_{\Delta}\right), & \delta_{\Delta}<X \leq \frac{\tau_{\Delta}}{2}\end{cases}$

If we set $X=\tau_{\Delta} / 2$ in this equation, we find after a little algebra and the use of Eq. (147) that we have, to within an exponentially small error,

$$
Q_{\Delta}^{\prime}\left(\tau_{\Delta}\right) \approx \frac{1}{2} \delta_{\Delta}\left(m-\tau_{\Delta}\right)
$$

However, the left-hand side must be precisely zero, since $\tau_{\Delta}$ is a local maximum of $Q_{\Delta}$. Since we have already shown that we must reject having $\delta_{\Delta}=o\left(\Delta^{3 / 2}\right)$, we must have

$$
m \approx \tau_{\Delta}+\text { exponentially small terms }
$$

and in all subsequent analysis we replace $m$ by $\tau_{\Delta}$. We can now conclude that from Eq. (164) that

$$
Q_{\Delta}^{\prime \prime}\left(\tau_{\Delta} / 2\right)=\tau_{\Delta}-1+\text { exponentially small terms. }
$$

Since we need $Q$ to have a minimum at $\tau_{\Delta} / 2$ we see the necessity of the restriction $\tau_{\Delta} \geq 1$, and we know from our earlier discussion that for any fixed $\Delta>0$ a non-constant solution with period $\tau_{\Delta}=1$ cannot exist.

From Eqs (161), (165) and (168) we conclude that

$$
\tau_{\Delta}^{2} \delta_{\Delta} \approx 2 \pi \Delta R_{\Delta}(0)^{2}
$$

This approximate equality essentially asserts that the asymptotic inequality (159), for which we have given a strong independent argument, holds with equality as $\Delta \rightarrow 0$. With $m \approx \tau_{\Delta}$ 
Eq. (166) becomes

$$
Q_{\Delta}^{\prime}\left(X+\frac{\tau_{\Delta}}{2}\right) \approx \begin{cases}\left(\tau_{\Delta}-1\right) X, & 0 \leq X<\delta_{\Delta}, \\ \left(\tau_{\Delta}-1\right) \delta_{\Delta}-\delta_{\Delta}\left(X-\delta_{\Delta}\right), & \delta_{\Delta}<X \leq \frac{\tau_{\Delta}}{2},\end{cases}
$$

and integrating again we have

$$
Q_{\Delta}\left(X+\frac{\tau_{\Delta}}{2}\right)-Q_{\Delta}\left(\frac{\tau_{\Delta}}{2}\right) \approx\left\{\begin{array}{l}
\frac{\left(\tau_{\Delta}-1\right) X^{2}}{2}, \quad 0 \leq X<\delta_{\Delta}, \\
\frac{\left(\tau_{\Delta}-1\right) \delta_{\Delta}^{2}}{2}+\left(\tau_{\Delta}-1\right) \delta_{\Delta}\left(X-\delta_{\Delta}\right) \\
-\frac{\delta_{\Delta}}{2}\left(X-\delta_{\Delta}\right)^{2}, \quad \delta_{\Delta}<X \leq \frac{\tau_{\Delta}}{2} .
\end{array}\right.
$$

In particular, setting $X=\tau_{\Delta} / 2$ and using $Q_{\Delta}(0)=Q_{\Delta}\left(\tau_{\Delta}\right)$, we find that

$$
Q_{\Delta}(0)-Q_{\Delta}\left(\tau_{\Delta} / 2\right) \approx \frac{\delta_{\Delta} \tau_{\Delta}\left(\tau_{\Delta}-1\right)}{4}
$$

and correspondingly

$$
\frac{R_{\Delta}(0)}{R_{\Delta}\left(\tau_{\Delta} / 2\right)} \approx \exp \left(\frac{\delta_{\Delta} \tau_{\Delta}\left(\tau_{\Delta}-1\right)}{4 \Delta}\right)
$$

We see once again, as we have already noted, that to stop the periodic solution degenerating to a constant as $\Delta \rightarrow 0$, we require $\delta_{\Delta} \neq o(\Delta)$ [that is, $\tau_{\Delta} \neq 2-o(\Delta)$ ] and also $\tau_{\Delta} \neq 1+o(\Delta)$.

We translate into the notation of the present section an important exact result from Section 6.4. Using the transformations $P(x)=(\beta /|\alpha|) R(X), x=\epsilon X$ and $\lambda=2 \pi /\left(\epsilon \tau_{\Delta}\right)$, we connect Fourier coefficients from the exact analysis of possible periodic solutions to our present approximate analysis based on a local Gaussian shape near the maxima. From Eq. (112),

$$
\log \left[\frac{\beta}{|\alpha|} R(X)\right]=\sum_{n=0}^{\infty} Q_{n} \cos \left(\frac{2 \pi n X}{\tau_{\Delta}}\right) .
$$

Hence from the orthogonality of the cosines,

$$
Q_{0}=\frac{1}{\tau_{\Delta}} \int_{0}^{\tau_{\Delta}} \log \left[\frac{\beta}{|\alpha|} R(X)\right] d X=\frac{2}{\tau_{\Delta}} \int_{\tau_{\Delta} / 2}^{\tau_{\Delta}} \log \left[\frac{\beta}{|\alpha|} R(X)\right] d X .
$$

The important $\Delta$-dependent quantity $R_{0}=(\beta /|\alpha|) \exp \left(Q_{0}\right)$ from Section 6.4, defined in Eq. (117), can now be calculated from the exact formula

$$
R_{0}=\exp \left[\frac{2}{\tau_{\Delta} \Delta} \int_{0}^{\tau_{\Delta} / 2} Q\left(X+\frac{\tau_{\Delta}}{2}\right) d X\right] .
$$

We insert the approximation (172) into Eq. (177) and find after some algebra, including the use of Eq. (173) and the relation $R_{\Delta}(0)=\exp \left[\Delta^{-1} Q_{\Delta}(0)\right]$, the approximation

$$
R_{0} \approx R_{\Delta}(0) \exp \left[-\frac{\left(\tau_{\Delta}-1\right)\left(2-\tau_{\Delta}\right)}{12 \Delta}\right]
$$

However, in the notation of the present section, the necessary and sufficient condition (130) for a 'string of delta functions limit' can be expressed as

$$
R_{0} \sim \frac{\tau_{\Delta}}{2 \pi^{1 / 2}}\left(\frac{2-\tau_{\Delta}}{\Delta}\right)^{1 / 2} \exp \left[-\frac{\left(\tau_{\Delta}-1\right)\left(2-\tau_{\Delta}\right)}{12 \Delta}\right]
$$

It is easy to see from Eq. (170) that the first factors on the right in Eqs (178) and (179) coincide. This correct alignment with the precise Fourier analytic requirements lends strong support to the assertion of the validity of the Gaussian approximations that we are using, the validity of the scaling law (170) and the existence of the 'string of delta functions' limit.

The scaling law (170) and our rigorously proved result (143) that $R_{\Delta}(0) \leq(e / \Delta)^{1 / 2}$ together lead to important conclusions about $\tau_{0}=\lim _{\Delta \rightarrow 0} \tau_{\Delta}$ :

(i) if $R_{\Delta}(0) \sim c \Delta^{-1 / 2}$ for some $c>0$ then $\tau_{0}<2$ : or

(ii) if $R_{\Delta}(0)=o\left(\Delta^{-1 / 2}\right)$ then $\tau_{0}=2$.

Numerical experiments performed by Hackett-Jones et al. [7] seem to suggest the second case. Although we have been unable to identify the value of $\tau_{0}$ analytically, there is a further way to check the overall consistency of the approach that we have followed. Details will be found in Appendix B.

\section{Equilibrium with nonlinear diffusion}

If we seek an equilibrium solution $\rho(x, t)=C P(x)$ in Eq. (17), where the constant $C$ is selected to ensure that $P(x)$ is correctly normalised as a probability density function, we have

$$
\mathrm{D}(C P) P^{\prime}(x)-C P(x) \int_{-\infty}^{\infty} f(x-\xi) P(\xi) d \xi=0 .
$$

Here we have dropped the Cauchy principal value notation, anticipating (as we shall find) that we are not troubled by slow decay of $P(x)$ as $|x| \rightarrow \infty$. This equation may be rewritten as

$$
\frac{d}{d x}\left\{\int \frac{\mathrm{D}(C P) d P}{P}+C \int_{-\infty}^{\infty} W(x-\xi) P(\xi) d \xi\right\}=0 .
$$

and we find on integrating that

$$
\int_{C P(x)}^{C P\left(\mu_{1}\right)} \frac{\mathrm{D}(\zeta) d \zeta}{\zeta}=C \int_{-\infty}^{\infty}\left[W(x-\xi)-W\left(\mu_{1}-\xi\right)\right] P(\xi) d \xi .
$$

\subsection{General observations}

If we assume that the potential $W$ is convex (this enforces $W(x) \rightarrow \infty$ as $|x| \rightarrow \infty)$, then using the consequence (36) of Jensen's inequality we have

$$
\int_{C P(x)}^{C P\left(\mu_{1}\right)} \frac{\mathrm{D}(\zeta) d \zeta}{\zeta} \geq C W\left(x-\mu_{1}\right)-C \int_{-\infty}^{\infty} W\left(\mu_{1}-\xi\right) P(\xi) d \xi
$$

We observe that if in addition we assume that $D(\rho) \rightarrow D_{0}$ as $\rho \rightarrow 0^{+}$, we conclude that $P(x) \rightarrow 0$ as $\left|x-\mu_{1}\right| \rightarrow \infty$ to enforce divergence of the integral on the left-hand side, and this divergence is as $D_{0} \log P(x)$, giving

$$
P(x) \leq \text { constant } \times \exp \left[-\left(C / D_{0}\right) W\left(x-\mu_{1}\right)\right] \text { for }\left|x-\mu_{1}\right| \rightarrow \infty .
$$

Comparing this bound with the inequality (37) for linear diffusion, we see that for nonlinear diffusion with $\mathrm{D}(\rho)$ bounded away from zero, the equilibrium solutions to not differ markedly 
in character from the case of classical linear diffusion, that is, constant $\mathrm{D}(\rho)$. However, if there are values of $\rho$ for which $\mathrm{D}(\rho)=0$, then the possibility of continuous, piecewise smooth solutions of Eq. (180) with compact support arises.

In general if $\mathrm{D}(\rho) \rightarrow 0$ as $\rho \rightarrow 0$, then a compact support solution symmetric about its mean $\mu_{1}$ consistent with Eq. (182) has $P(x)=0$ for $\left|x-\mu_{1}\right| \geq L$, while for $\left|x-\mu_{1}\right|<L$,

$$
\int_{C P(x)}^{C P\left(\mu_{1}\right)} \frac{\mathrm{D}(\zeta) d \zeta}{\zeta}=C \int_{\mu_{1}-L}^{\mu_{1}+L}\left[W(x-\xi)-W\left(\mu_{1}-\xi\right)\right] P(\xi) d \xi
$$

Here

$$
\int_{0}^{C P\left(\mu_{1}\right)} \frac{\mathrm{D}(\zeta) d \zeta}{\zeta}=C \int_{\mu_{1}-L}^{\mu_{1}+L}\left[W( \pm L-\xi)-W\left(\mu_{1}-\xi\right)\right] P(\xi) d \xi
$$

and

$$
\int_{\mu_{1}-L}^{\mu_{1}+L} P(x) d x=1 .
$$

A specific illustration of this is given in Section 7.2.

\subsection{Quadratic cohesive potential}

For nonlinear diffusion with the cohesive quadratic potential $W(x)=\beta x^{2}$ (with $\beta>0$ ), we find (using the special case of Eq. (59) corresponding to $\delta=0$ ) that

$$
\int_{C P(x)}^{C P\left(\mu_{1}\right)} \frac{\mathrm{D}(\rho) d \rho}{\rho}=\beta C\left(x-\mu_{1}\right)^{2} .
$$

Some quite strong conclusions can now be drawn: $P(x)$ is symmetric, is monotonic with respect to $\left|x-\mu_{1}\right|$, and if $\mathrm{D}(\rho) \geq D_{0}$ with $\mathrm{D}(\rho) \rightarrow D_{0}$ as $\rho \rightarrow 0^{+}$, then we have

$$
P(x) \sim P\left(\mu_{1}\right) \exp \left[-\left(\beta C / D_{0}\right)\left(x-\mu_{1}\right)^{2}\right] \text { as }\left|x-\mu_{1}\right| \rightarrow \infty .
$$

However, if $\mathrm{D}(0)=0$, with $\mathrm{D}(\rho)$ vanishing fast enough as $\rho \rightarrow$ $0^{+}$to ensure that $D(\rho) / \rho$ has a finite improper Riemann integral integral on $(0, \epsilon)$ for $\epsilon>0$, we can satisfy Eq. (180) by taking $P(x) \equiv 0$ for $\left|x-\mu_{1}\right| \geq L$, and

$$
\int_{C P(x)}^{C P\left(\mu_{1}\right)} \frac{\mathrm{D}(\rho) d \rho}{\rho}=\beta C\left(x-\mu_{1}\right)^{2} \quad \text { for }\left|x-\mu_{1}\right|<L,
$$

with $L$ and $P\left(\mu_{1}\right)$ chosen so that

$$
\int_{0}^{C P\left(\mu_{1}\right)} \frac{\mathrm{D}(\rho) d \rho}{\rho}=\beta C L^{2}, \quad \int_{\mu_{1}-L}^{\mu_{1}+L} P(x) d x=1 .
$$

This demonstrates that compact support equilibrium solutions arise for some nonlinear diffusivities.

\subsection{Power-law diffusivity}

The so-called porous medium equation $\partial u / \partial t=\nabla^{2}\left(u^{m}\right)$ with $m>1$ is equivalent to a power-law diffusivity, and we use this an an illustration. If we take

$$
\mathrm{D}(\rho)=D_{\eta} \rho^{\eta}
$$

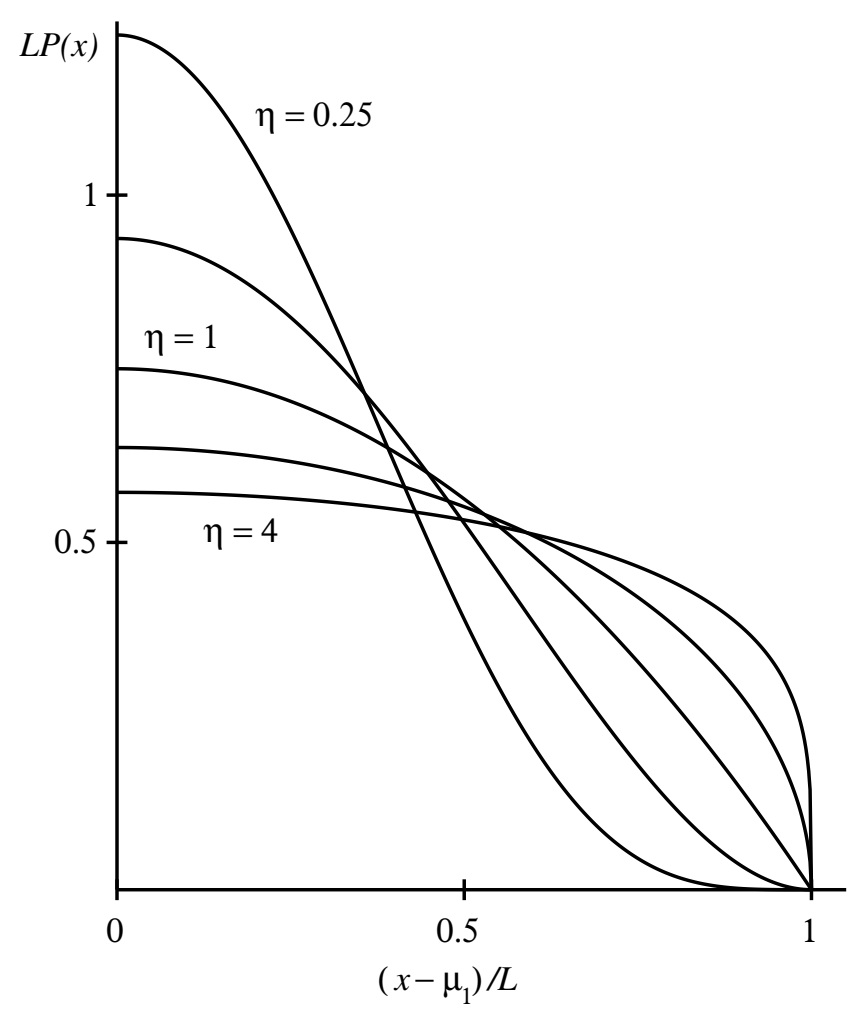

Figure 5: The equilibrium probability density function $P(x)$ for the power-law diffusivity $\mathrm{D}(\rho)=D_{\eta} \rho^{\eta}$ and the quadratic cohesive potential $W(x)=\beta x^{2}$. The half-width $L$ of the support of the density is given by Eq. (196).

with $\eta$ a positive constant, then we find that for the quadratic cohesive potential $W(x)=\beta x^{2}$,

$$
P(x)=\left\{\frac{\eta \beta C^{1-\eta}}{D_{\eta}}\left[L^{2}-\left(x-\mu_{1}\right)^{2}\right]\right\}^{1 / \eta}, \quad 0 \leq\left|x-\mu_{1}\right| \leq L .
$$

where for normalisation we require

$$
\int_{0}^{L}\left\{\frac{\eta \beta C^{1-\eta}}{D_{\eta}}\left[L^{2}-x^{2}\right]\right\}^{1 / \eta} d x=\frac{1}{2} .
$$

The integral is easily evaluated in terms of the beta function $B(u, v)=\Gamma(u) \Gamma(v) / \Gamma(u+v)$ and we find that

$$
P(x)=\frac{1}{L B(1 / 2,1 / \eta+1)}\left[1-\frac{\left(x-\mu_{1}\right)^{2}}{L^{2}}\right]^{1 / \eta}, \quad\left|x-\mu_{1}\right| \leq L,
$$

where $L$ is given by

$$
L^{1+2 / \eta}\left[\frac{\eta \beta C^{1-\eta}}{D_{\eta}}\right]^{1 / \eta} B(1 / 2,1 / \eta+1)=1 .
$$

We show $L P(x)$ as a function of $\left|x-\mu_{1}\right| / L$ in Fig. 5 .

An analysis of the equilibrium problem for a power-law diffusivity (192) and the more general potential $W(x)=\alpha|x|+\beta x^{2}$ will be found in Appendix $\mathrm{C}$.

\section{Time evolution with a quadratic cohesive potential}

From now on we consider only the case

$$
f(x)=-2 \beta x,
$$


corresponding to the quadratic cohesive interparticle potential $W(x)=\beta x^{2}$. In this case

$$
\begin{aligned}
\frac{\partial p}{\partial t} & =\mathcal{D} p(x, t)+2 \beta C \frac{\partial}{\partial x}\left\{p(x, t) \int_{-\infty}^{\infty}(x-\xi) p(\xi, t) d \xi\right\} \\
& =\mathcal{D} p(x, t)+2 \beta C \frac{\partial}{\partial x}\{p(x, t)[x-\mu(t)]\},
\end{aligned}
$$

where

$$
\mu(t)=\int_{-\infty}^{\infty} x p(x, t) d x
$$

is the mean position if the integral is absolutely convergent. If the integral is not absolutely convergent (so that it must not be interpreted as a mean), the function $\mu(t)$ will still exist in the principal value sense, and indeed be zero, if $p(x, t)$ is an even function of $x$. In this section we discuss the time evolution of $P(x, t)$ in a context somewhat more general than classical diffusion.

\subsection{Classical time variation}

We defer for the moment the general case in which the operator $\mathcal{D}$ may act on the temporal variable as well as the space variable, so that we are looking at the cases designated (a) and (b) in Section 2. Taking a Fourier transform and assuming appropriate decay of the solution as $|x| \rightarrow \infty$, from Eq. (199) we have

$$
\begin{aligned}
\frac{\partial \tilde{p}}{\partial t} & =-a(q) \tilde{p}(q, t)-2 \beta C i q \int_{-\infty}^{\infty} e^{i q x} p(x, t)[x-\mu(t)] d x \\
& =-a(q) \tilde{p}(q, t)-2 \beta C i q\left[-i \frac{\partial \tilde{p}}{\partial q}-\mu(t) \tilde{p}\right]
\end{aligned}
$$

leading to the linear first-order partial differential equation

$$
\frac{\partial \tilde{p}}{\partial t}+2 \beta C q \frac{\partial \tilde{p}}{\partial q}=-[a(q)-2 \beta C i q \mu(t)] \tilde{p}(q, t) .
$$

To solve Eq. (203) we first write

$$
\tilde{p}(q, t)=\exp \left\{\chi(q, t)-\frac{1}{2 \beta C} \int_{0}^{q} \frac{a(s) d s}{s}\right\},
$$

and we deduce that

$$
\frac{\partial \chi}{\partial t}+2 \beta C q \frac{\partial \chi}{\partial q}=2 \beta C i q \mu(t)
$$

The characteristics for this first-order partial differential equation are given by

$$
\frac{d q}{d t}=2 \beta C q
$$

corresponding to $\xi=q \exp (-2 \beta C t)$ being constant on a characteristic. If we write

$$
\chi(q, t)=F(q \exp (-2 \beta C t), t)
$$

we find that

$$
\frac{\partial F}{\partial t}=2 \beta C i \xi e^{2 \beta C t} \mu(t)
$$

Integrating (and remembering to add on an arbitrary function of $\xi$ in so doing), we find after a little algebra that the solution for $\tilde{p}(q, t)$ has the form

$$
\begin{aligned}
\tilde{p}(q, t) & =Q\left(q e^{-2 \beta C t}\right) \\
& \times \exp \left\{2 \beta C i q \int_{0}^{t} e^{-2 \beta C(t-\tau)} \mu(\tau) d \tau-\frac{1}{2 \beta C} \int_{0}^{q} \frac{a(s) d s}{s}\right\},
\end{aligned}
$$

where the function $Q$ is determined by the initial conditions. Setting $t=0$ we see that

$$
\tilde{p}(q, 0)=Q(q) \exp \left\{-\frac{1}{2 \beta C} \int_{0}^{q} \frac{a(s) d s}{s}\right\}
$$

and so our solution for the Fourier transform of the probability density function becomes

$$
\begin{aligned}
\tilde{p}(q, t)= & \tilde{p}\left(q e^{-2 \beta C t}, 0\right) \\
& \times \exp \left\{2 \beta C i q \int_{0}^{t} e^{-2 \beta C(t-\tau)} \mu(\tau) d \tau-\frac{1}{2 \beta C} \int_{q e^{-2 \beta C t}}^{q} \frac{a(s) d s}{s}\right\} .
\end{aligned}
$$

Let us assume for the moment that the mean $\mu(t)$ exists in the classical sense as an absolutely convergent integral. Since $\tilde{p}(0, t)=1$ and

$$
\int_{\infty}^{\infty} x p(x, t) d x=-\left.i \frac{\partial}{\partial q} \tilde{p}(q, t)\right|_{q=0}
$$

we see that

$$
\mu(t)=-\left.i \frac{\partial}{\partial q} \log [\tilde{p}(q, t)]\right|_{q=0}
$$

and we find that

$$
\begin{aligned}
\mu(t)= & \mu(0) \exp (-2 \beta C t)+2 \beta C \int_{0}^{t} \exp [-2 \beta C(t-\tau)] \mu(\tau) d \tau \\
& +\frac{i}{2 \beta C} \lim _{q \rightarrow 0} \frac{a(q)-a(q \exp (-2 \beta C t))}{q}
\end{aligned}
$$

For classical diffusion with a constant drift velocity $v$ we have $a(q)=D q^{2}-i v q$, and more generally if we have $a(q)=-i v q+$ $o(q)$ as $q \rightarrow 0$ we are able to deduce that

$$
\begin{aligned}
\mu(t)= & \mu(0) \exp (-2 \beta C t)+2 \beta C \int_{0}^{t} \exp [-2 \beta C(t-\tau)] \mu(\tau) d \tau \\
& \left.+\frac{v}{2 \beta C}[1-\exp (-2 \beta C t))\right]
\end{aligned}
$$

This integral equation can be solved using Laplace transforms. We have, on using the convolution theorem,

$$
\widehat{\mu}(u)=\frac{\mu(0)}{u+2 \beta C}+\frac{2 \beta C \widehat{\mu}(u)}{u+2 \beta C}+\frac{v}{2 \beta C}\left[\frac{1}{u}-\frac{1}{u+2 \beta C}\right] .
$$

From this we find that $\widehat{\mu}(u)=u^{-1} \mu(0)+u^{-2} v$, whence we extract the not surprising result that

$$
\mu(t)=\mu(0)+v t
$$


Thus in general if we have $a(q)=o(q)$ as $q \rightarrow 0$, corresponding to $v=0$, we find that $\mu(t)=\mu(0)$ for all $t \geq 0$.

Can we obtain a sensible evolution equation for $\mu(t)$ when the integral that defines $\mu(t)$ exists only as a principal value integral? We have

$$
\begin{aligned}
\mu(t) & =\int_{-\infty}^{\infty} x p(x, t) d x=\lim _{L \rightarrow \infty} \int_{-L}^{L} x p(x, t) d x \\
& =\lim _{L \rightarrow \infty} \int_{-L}^{L} \frac{x}{2 \pi} \int_{-\infty}^{\infty} e^{-i q x} \tilde{p}(q, t) d q d x \\
& =\lim _{L \rightarrow \infty} \int_{-\infty}^{\infty} \frac{\tilde{p}(q, t)}{2 \pi} \int_{-L}^{L} x e^{-i q x} d x d q \\
& =\frac{i}{\pi} \lim _{L \rightarrow \infty} \int_{-\infty}^{\infty} \tilde{p}(q, t) \frac{\partial}{\partial q} \frac{\sin (q L)}{q} d q \\
& =-\frac{i}{\pi} \lim _{L \rightarrow \infty} \int_{-\infty}^{\infty} \frac{\sin (q L)}{q} \frac{\partial}{\partial q} \tilde{p}(q, t) d q
\end{aligned}
$$

Using the standard limit

$$
\lim _{L \rightarrow \infty} \int_{0}^{\infty} \frac{\sin (q L)}{q} f(q) d q=\frac{\pi}{2} \lim _{q \rightarrow 0^{+}} f(q),
$$

which holds under modest continuity and integrability conditions on $f$, we find that

$$
\mu(t)=\frac{i}{2}\left[\lim _{q \rightarrow 0-} \frac{\partial}{\partial q} \tilde{p}(q, t)+\lim _{q \rightarrow 0+} \frac{\partial}{\partial q} \tilde{p}(q, t)\right]
$$

and unless we have $a(q)=O(q)$ (or smaller) as $q \rightarrow 0$, the righthand side fails to exist. We shall assume henceforth that $a(q)=$ $O(q)$ as $q \rightarrow 0$. Moreover, in the case in which $a(q) \sim-i v q$ as $q \rightarrow 0$, we get a simple drift in the mean position, so there is no serious loss of generality in taking $v=0$ and considering only cases in which the initial mean is $\mu(0)=0$, the mean remains zero for all time, and $a(q)=o(q)$.

Setting $\mu(t)=0$ for all $t \geq 0$ in Eq. (211), we have

$$
\tilde{p}(q, t)=\tilde{p}\left(q e^{-2 \beta C t}, 0\right) \exp \left\{-\frac{1}{2 \beta C} \int_{q e^{-2 \beta C t}}^{q} \frac{a(s) d s}{s}\right\} .
$$

Since $\tilde{p}(q, 0)$ is the Fourier transform of a probability density function, it is necessarily a continuous function of $q$. It follows that as $t \rightarrow \infty$,

$$
\tilde{p}\left(q e^{-2 \beta C t}, 0\right) \rightarrow \tilde{p}(0,0)=\int_{-\infty}^{\infty} p(x, 0) d x=1 .
$$

Hence

$$
\lim _{t \rightarrow \infty} \tilde{p}(q, t)=\exp \left\{-\frac{1}{2 \beta C} \int_{0}^{q} \frac{a(s) d s}{s}\right\}
$$

and we find that we have a well-defined long-term limiting probability density function. In particular, if we take $a(q)=K|q|^{K}$ $(0<\kappa \leq 2)$, so that the system would evolve as a symmetric stable process of order $\kappa$ if the cohesive potential were turned off $(\beta=0)$, we find that

$$
\lim _{t \rightarrow \infty} \tilde{p}(q, t)=\exp \left\{-\frac{K|q|^{k}}{2 \kappa \beta C}\right\}
$$

corresponding to the equilibrium distribution also being a stable law of order $\kappa$.

To investigate the time-dependence of the solution, we invert the Fourier transform in Eq. (221) using the convolution theorem, finding that

$$
p(x, t)=\int_{-\infty}^{\infty} A(\xi, t) B(x-\xi, t) d \xi
$$

where

$$
\tilde{A}(q, t)=\tilde{p}\left(q e^{-2 \beta C t}, 0\right), \quad \tilde{B}(q, t)=\exp \left\{-\frac{1}{2 \beta C} \int_{q e^{-2 \beta C t}}^{q} \frac{a(s) d s}{s}\right\},
$$

so that

$$
\begin{aligned}
A(x, t) & =\frac{1}{2 \pi} \int_{-\infty}^{\infty} e^{-i q x} \tilde{p}\left(q e^{-2 \beta C t}, 0\right) d q \\
& =e^{2 \beta C t} \frac{1}{2 \pi} \int_{-\infty}^{\infty} \exp \left(-i q x e^{2 \beta C t}\right) \tilde{p}(q, 0) d q \\
& =e^{2 \beta C t} p\left(x e^{2 \beta C t}, 0\right) .
\end{aligned}
$$

The function $B(x, t)$ can be interpreted as the response of the system given the initial condition $p(x, 0)=\delta(x)$. It is easy to see that if $a(s)$ is an even function, then $\tilde{B}(q, t)$ is an even function of $q$. In particular if $a(q)=K|q|^{\kappa}$ with $\kappa \in(1,2]$, we have

$$
\tilde{B}(q, t)=\exp \left\{-\frac{K}{2 \kappa \beta C}\left[1-e^{-2 \kappa \beta C t}\right]|q|^{\kappa}\right\}
$$

so that at each time $t, B(x, t)$ is a stable density of order $\kappa$. If we recall that

$$
\mathcal{F}\left\{\frac{1}{\sqrt{4 \pi D t}} \exp \left(-\frac{x^{2}}{4 D t}\right) ; x \mapsto q\right\}=\exp \left(-D q^{2}\right),
$$

we see that when the underlying transport process is ordinary symmetric diffusion, that is,

$$
\mathcal{D}=D \frac{\partial^{2}}{\partial x^{2}} \quad \text { and } \quad a(q)=D q^{2}
$$

we have

$$
B(x, t)=\sqrt{\frac{\beta C}{\pi D\left(1-e^{-4 \beta C t}\right)}} \exp \left[-\frac{\beta C x^{2}}{D\left(1-e^{-4 \beta C t}\right)}\right]
$$

and we observe the limiting behaviours

$$
\begin{aligned}
& B(x, t) \sim \frac{1}{\sqrt{4 \pi D t}} \exp \left(-\frac{x^{2}}{4 D t}\right) \quad \text { as } t \rightarrow 0^{+}, \\
& B(x, t) \rightarrow \sqrt{\frac{\beta C}{\pi D}} \exp \left(-\frac{\beta C x^{2}}{D}\right) \quad \text { as } t \rightarrow \infty .
\end{aligned}
$$

We note that most of our preceding discussion in this section covers quite general choices of $a(q)$, though we have used as illustrations the cases when the motility mechanism is linear diffusion or a stable process. Biler and Karch [44] have given some related results for the specific case of stable processes, when the cohesive potential $W$ is a single-component powerlaw potential $W$. 


\subsection{Fractional diffusion models}

Other transport processes can of course be considered, including fractional diffusion processes that have become somewhat popular in the last decade or so [32, 43]. For example, one may consider [45] the fractional diffusion equation

$$
{ }_{t} D_{+}^{\lambda} p(x, t)=K_{x} D_{0}^{\kappa} p(x, t), \quad 0<\kappa \leq 2, \quad 0<\lambda \leq 1, \quad K>0,
$$

which reduces to the standard diffusion equation with diffusivity $K$ if $\kappa=2$ and $\lambda=1$, but corresponds to an integrodifferential equation in the remaining cases. Here for $0<\lambda<1$ the Caputo time derivative of fractional order $\lambda$ is defined by

$$
{ }_{t} D_{+}^{\lambda} f(t)=\frac{1}{\Gamma(1-\lambda)} \int_{0}^{t} \frac{f^{\prime}(\tau) d \tau}{(t-\tau)^{\lambda}},
$$

while for $0<\kappa<2$ the Riesz fractional derivative is defined by

${ }_{x} D_{0}^{\kappa} g(x)=\frac{\Gamma(1+\kappa) \sin (\pi \kappa / 2)}{\pi} \int_{0}^{\infty} \frac{g(x+\xi)-2 g(x)+g(x-\xi)}{\xi^{1+\kappa}}$.

It is easy to show that

$$
\mathcal{L}\left\{{ }_{t} D_{+}^{\lambda} f(t) ; t \rightarrow u\right\}=u^{\lambda} \mathcal{L}\{f(t) ; t \rightarrow u\}-u^{\lambda-1} \lim _{t \rightarrow 0^{+}} f(t),
$$

$$
\mathcal{F}\left\{{ }_{x} D_{0}^{\kappa} g(x) ; x \rightarrow q\right\}=-|q|^{\kappa} \mathcal{F}\{g(x) ; x \rightarrow q\},
$$

consistent with the familiar $\lambda=1$ and $\kappa=2$ cases

$$
\begin{aligned}
\mathcal{L}\left\{f^{\prime}(t) ; t \rightarrow u\right\} & =u \mathcal{L}\{f(t) ; t \rightarrow u\}-\lim _{t \rightarrow 0^{+}} f(t), \\
\mathcal{F}\left\{g^{\prime \prime}(x) ; x \rightarrow q\right\} & =-|q|^{2} \mathcal{F}\{g(x) ; x \rightarrow q\}
\end{aligned}
$$

Note however that for $\kappa=1$ we do not recover the standard classical result that

$$
\mathcal{F}\left\{g^{\prime}(x) ; x \rightarrow q\right\}=-i q \mathcal{F}\{g(x) ; x \rightarrow q\}
$$

If we take the joint Fourier-Laplace transform of the fractional diffusion equation (234) we find that

$$
u^{\lambda} p^{*}(q, u)-u^{\lambda-1} \tilde{p}(q, 0)=-K|q|^{\kappa} p^{*}(q, u)
$$

To make this look as much as possible like a diffusion process, we rewrite the equation as [46]

$$
u p^{*}(q, u)-\tilde{p}(q, 0)=-K u^{1-\lambda}|q|^{\kappa} p^{*}(q, u) .
$$

and we recognize this as the Laplace transform of the equation

$$
\frac{\partial}{\partial t} \tilde{p}(q, t)=-\frac{K}{\Gamma(\lambda)} \frac{\partial}{\partial t} \int_{0}^{t} \frac{|q|^{K} \tilde{p}\left(q, t^{\prime}\right) d t^{\prime}}{\left(t-t^{\prime}\right)^{1-\lambda}} .
$$

If we take this equation as our underlying transport process and add in the cohesive force effect, we have an example of the third class of models discussed in the introduction [case (c)] in which

$$
\begin{aligned}
\mathcal{J}\{\mathcal{D} p(x, t) ; x \rightarrow q, t \rightarrow u\} & =\int_{-\infty}^{\infty} e^{i q x} \int_{0}^{\infty} e^{-u t} \mathcal{D} p(x, t) d t d x \\
& =-b(q, u) p^{*}(q, u),
\end{aligned}
$$

with

$$
b(q, u)=K u^{1-\lambda}|q|^{K} .
$$

We note in passing that the modification of fractional diffusion processes to include additional effects requires some care. For example, when reaction terms that remove mass are added, although solutions remain non-negative in the limiting case of normal diffusion, in the genuinely factional case, solutions can be come unphysically negative unless the way in which the reaction terms are incorporated is done very carefully [51]. However, as we combine two flux mechanisms, each of which conserves mass, we anticipate no such problems here, although we do not prove non-negativity of solutions.

For the general model of case (c), to ensure that $\mu(t)$ is well-defined and zero for all times $t$, we shall require $\tilde{p}(q, 0)$ and $b(q, s)$ to be even functions of $q$. We have from the joint Fourier-Laplace transform of the evolution equation

$$
u p^{*}(q, u)-\tilde{p}(q, 0)=-b(q, u) p^{*}(q, u)-2 \beta C q \frac{\partial}{\partial q} p^{*}(q, u) .
$$

This can be written in standard form as a first-order linear differential equation for $p^{*}(q, u)$ :

$$
\frac{\partial}{\partial q} p^{*}(q, u)+\left[\frac{u}{2 \beta C q}+\frac{b(q, u)}{2 \beta C q}\right] p^{*}(q, u)=\frac{\tilde{p}(q, 0)}{2 \beta C q} .
$$

Multiplying both sides of this equation by an appropriate integrating factor, and fitting the arbitrary function of $u$ that arises in performing the integration, we find that

$$
\begin{aligned}
p^{*}(q, u) & =\int_{0}^{|q|} \frac{\tilde{p}(r, 0)}{2 \beta C r}\left(\frac{r}{|q|}\right)^{u /(2 \beta C)} \exp \left\{-\int_{r}^{|q|} \frac{b(s, u) d s}{2 \beta C s}\right\} d r \\
& =\int_{0}^{1} \frac{\tilde{p}(|q| \xi, 0)}{2 \beta C} \xi^{u /(2 \beta C)-1} \exp \left\{-\int_{|q| \xi}^{|q|} \frac{b(s, u) d s}{2 \beta C s}\right\} d \xi .
\end{aligned}
$$

What can we deduce from this equation by asymptotic analysis? As $q \rightarrow 0$ the left-hand side converges to

$$
p^{*}(0, u)=\mathcal{L}\left\{\int_{-\infty}^{\infty} p(x, t) d x ; t \mapsto u\right\}=\mathcal{L}\{1 ; t \mapsto u\}=\frac{1}{u}
$$

In the right-hand side

$$
\tilde{p}(|q| \xi, 0) \rightarrow \int_{-\infty}^{\infty} p(x, t) d x=1,
$$

so the right-hand side converges to

$$
\int_{0}^{1} \frac{1}{2 \beta C} \xi^{u /(2 \beta C)-1} d \xi=\frac{1}{u}
$$

also, and so we have verified correct normalization for all times.

Now we hold $q$ constant and let $u \rightarrow 0$ to try to extract the long-time behaviour. We shall assume that $b(q, u) \rightarrow 0$ as $u \rightarrow 0^{+}$. This is the case for the fractional diffusion operator, 
from Eq. (246). We see from Eq. (250) that as $u \rightarrow 0^{+}$, the argument of the exponential function vanishes and

$$
\begin{aligned}
p^{*}(q, u) & \sim \int_{0}^{1} \frac{\tilde{p}(|q| \xi, 0)}{2 \beta C} \xi^{u /(2 \beta C)-1} d \xi \\
& =\frac{1}{u} \int_{0}^{1} \tilde{p}(|q| \xi, 0) \frac{d}{d \xi} \xi^{u /(2 \beta C)} d \xi \\
& =\frac{1}{u}\left\{\left[\tilde{p}(|q| \xi, 0) \xi^{u /(2 \beta C)}\right]_{0}^{1}-\int_{0}^{1} \xi^{u /(2 \beta C)} \frac{d}{d \xi} \tilde{p}(|q| \xi, 0) d \xi\right\} \\
& \sim \frac{1}{u}\left\{\tilde{p}(|q|, 0)-\int_{0}^{1} \frac{d}{d \xi} \tilde{p}(|q| \xi, 0) d \xi\right\}=\frac{\tilde{p}(0,0)}{u} \\
& =\frac{1}{u} \int_{-\infty}^{\infty} p(x, 0) d x=\frac{1}{u} .
\end{aligned}
$$

Since the asymptotic behaviour of a function $f(t)$ as $t \rightarrow \infty$ is captured in the behaviour of its Laplace transform $\widehat{f}(u)$ as $u \rightarrow 0^{+}$, we see that as $t \rightarrow \infty, \tilde{p}(q, t) \rightarrow 1$. Recall that we have denoted Dirac's delta function by $\delta(\cdot)$ to distinguish it from the parameter $\delta$ used earlier. Since $\tilde{p}(q, t) \rightarrow 1$ as $t \rightarrow \infty$, we conclude that $p(x, t) \rightarrow \delta(x)$. That is, the process become localized at the origin. This conclusion holds in all cases where $p(x, 0)$ is an even function and the mean location exists, provided that $b(q, u) \rightarrow 0$ as $b \rightarrow 0^{+}$(and so in particular for the case (246) with $0<\lambda<1$ and $1<\kappa \leq 2$ ).

\section{Conclusions}

We have show in this paper how a continuous swarm of agents subject to the combination of a local motility mechanism reflecting random motion and a local drift velocity computed from long-range, ultimately cohesive forces between agents, evolves over time and we have paid particular attention to possible equilibrium states relevant to long-time limiting behaviour. Our results embrace a wide variety of motility mechanisms, and include some results for general long-range interactions, but also many exact analytic results for special nontrivial forms of the long-range interaction. In the particular case where the motility mechanism is standard linear diffusion, we have been able to probe the limiting behaviour of equilibrium solutions as the diffusivity tends to zero, shedding light on some puzzling aspects of purely deterministic long-range interaction models, especially the genesis of closely-spaces spikes of concentration, apparently converging to Dirac delta functions.

All of our results have been for processes with the real line $\mathbb{R}$ as the space domain. Many of the same ideas may be developed in $\mathbb{R}^{2}$ and $\mathbb{R}^{3}$, although the possible behaviours of smoothed singular potentials in the low-diffusivity limit may be somewhat more exotic than the scenarios for which we have given evidence in one space dimension in Section 6.

All our results have been for a continuous swarm of interacting agents. It is reasonable to expect that if $N \gg 1$ an appropriately defined swarm of $N$ discrete individuals will behave the same way. For such a swarm, the $j$ th individual has location $X_{j}(t)$ at time $t$ and contributes a mass $\delta\left(x-X_{j}(t)\right)$ to the overall mass density from which the ultimately cohesive drift velocity is computed. The motility of the individual is described by the sum of displacement under the drift velocity and random displacement appropriate to sample paths of the random process underlying the motility operator $\mathcal{D}$. Note that a rigorous construction of the discrete system may not be possible for nonlinear diffusion processes, as there appears to be lacking any general exact correspondence between nonlinear diffusion and an underlying stochastic process, although connections can be made within a mean field approximation. With that caveat, the interplay between motility rules that promote or suppress local clustering, and the long-range forces, that may have repulsive regimes as well as dominant cohesion in the far field, could turn out to be subtle and interesting.

\section{Acknowledgement}

We thank Professor Kerry Landman and Dr Emily HackettJones for helpful discussions, Dr Gaël Raoul for drawing several references to our attention, and the referees for useful comments.

\section{Appendix A. Asymptotic analysis for linear diffusion with $D \rightarrow 0$ and potential $W(x)=-|\alpha| x+\beta x^{2}$}

We rewrite Eq. (82) as

$$
1=\frac{P\left(\mu_{1}\right) D^{1 / 2}}{(|\alpha| C)^{1 / 2}} \int_{0}^{1} \frac{d p}{\left\{\left[\frac{\beta}{|\alpha|}-P\left(\mu_{1}\right)\right](1-p)+\frac{\beta}{|\alpha|} Q(p)\right\}^{1 / 2}}
$$

where

$$
Q(p)=\log (1 / p)-(1-p) \sim \frac{(1-p)^{2}}{2} \text { as } p \rightarrow 1^{-} .
$$

For correct normalization, we need the integral to diverge appropriately as $D \rightarrow 0$ and this can only happen if $P\left(\mu_{1}\right) \rightarrow \beta /|\alpha|$ in an appropriate way which we shall determine. For brevity we set $S=\beta /|\alpha|-P\left(\mu_{1}\right)$ and write

$$
\begin{aligned}
& I(S)=\int_{0}^{1} \frac{d p}{\left\{\left[S(1-p)+\frac{\beta}{|\alpha|} Q(p)\right\}^{1 / 2}\right.}, \\
& J(S)=\int_{0}^{1} \frac{d p}{\left\{\left[S(1-p)+\frac{\beta}{|\alpha|} \frac{(1-p)^{2}}{2}\right\}^{1 / 2}\right.} .
\end{aligned}
$$

We require the dominant small- $S$ asymptotic behaviour of $I(S)$. However, it is straightforward to show that $I(S)-J(S)$ converges to a constant as $S \rightarrow 0^{+}$, so it suffices to consider the dominant asymptotic behaviour of the simpler integral $J(S)$. This integral is elementary:

$$
J(S)=\left(\frac{2}{\beta}\right)^{1 / 2} \log \left[\frac{\beta}{S}+\sqrt{\left(\frac{\beta}{S}\right)^{2}+1}\right] \sim\left(\frac{2}{\beta}\right)^{1 / 2} \log \left(\frac{2 \beta}{S}\right) .
$$

If we set

$$
S=\beta /|\alpha|-P\left(\mu_{1}\right)=K \exp \left[-\kappa D^{-1 / 2}\right],
$$


then the value of the positive constant $K$ is not determined, but we find that the divergence of the integral preserves the normalization if and only if

$$
\kappa=\left(\frac{C \alpha^{2}}{2 \beta}\right)^{1 / 2} .
$$

Thus we have established Eq. (83).

\section{Appendix B. Consistency of the asymptotic arguments in Section 6.5}

We shall compute the period $\tau_{\Delta}$ on the basis of the approximate formulae that we have already obtained. We have

$$
\begin{aligned}
\frac{\tau_{\Delta}}{2} & =\int_{0}^{\tau_{\Delta} / 2} R_{\Delta}\left(\frac{\tau_{\Delta}}{2}+X\right) d X \\
& =R_{\Delta}\left(\frac{\tau_{\Delta}}{2}\right) \int_{0}^{\tau_{\Delta} / 2} \exp \left\{\frac{1}{\Delta}\left[Q\left(\frac{\tau_{\Delta}}{2}+X\right)-Q\left(\frac{\tau_{\Delta}}{2}\right)\right]\right\} d X .
\end{aligned}
$$

We insert the approximation (172) into the integrand and find (noting that $\tau_{\Delta} / 2-\delta_{\Delta}=\tau_{\Delta}-1$ ) that

$$
\begin{aligned}
\frac{\tau_{\Delta}}{2} \approx & R_{\Delta}\left(\frac{\tau_{\Delta}}{2}\right) \int_{0}^{\delta_{\Delta}} \exp \left[\frac{\left(\tau_{\Delta}-1\right) X^{2}}{2 \Delta}\right] d X+R_{\Delta}\left(\frac{\tau_{\Delta}}{2}\right) \\
& \times \int_{0}^{\tau_{\Delta}-1} \exp \left[\frac{\left(\tau_{\Delta}-1\right) \delta_{\Delta}^{2}}{2 \Delta}+\frac{\left(\tau_{\Delta}-1\right) \delta_{\Delta} X}{\Delta}-\frac{\delta_{\Delta} X^{2}}{2 \Delta}\right] d X .
\end{aligned}
$$

Next, we complete the square in the second integral and change the integration variable from $X$ to $\tau_{\Delta}-1-X$, and we eliminate $R_{\Delta}\left(\tau_{\Delta} / 2\right)$ in favour of $R_{\Delta}(0)$ using Eq. (174), giving after a little algebra

$$
\begin{gathered}
\frac{\tau_{\Delta}}{2} \approx R_{\Delta}(0) \exp \left[-\frac{\delta_{\Delta} \tau_{\Delta}\left(\tau_{\Delta}-1\right)}{4 \Delta}\right] \int_{0}^{\delta_{\Delta}} \exp \left[\frac{\left(\tau_{\Delta}-1\right) X^{2}}{2 \Delta}\right] d X \\
+R_{\Delta}(0) \int_{0}^{\tau_{\Delta}-1} \exp \left(-\frac{\delta_{\Delta} X^{2}}{2 \Delta}\right) d X
\end{gathered}
$$

The second integral in Eq. (B.2) can be expressed in terms of the usual error function [47]

$\operatorname{erf}(z)=\frac{2}{\sqrt{\pi}} \int_{0}^{z} e^{-x^{2}} d x= \begin{cases}\frac{2}{\sqrt{\pi}}\left[z+O\left(z^{3}\right)\right] & \text { as } z \rightarrow 0^{+} \\ 1-\frac{e^{-z^{2}}}{z \sqrt{\pi}}\left[1+O\left(z^{-2}\right)\right] & \text { as } z \rightarrow \infty\end{cases}$

while it is useful to extract the maximum value of the integrand as a factor from the first integral. Then Eq. (B.2) becomes

$$
\begin{gathered}
\frac{\tau_{\Delta}}{2} \approx R_{\Delta}(0) \exp \left[-\frac{\delta_{\Delta}\left(\tau_{\Delta}-1\right)^{2}}{2 \Delta}\right] \int_{0}^{\delta_{\Delta}} \exp \left[-\frac{\left(\tau_{\Delta}-1\right)\left(\delta_{\Delta}^{2}-X^{2}\right)}{2 \Delta}\right] d X \\
+\frac{R_{\Delta}(0)}{2}\left(\frac{2 \pi \Delta}{\delta_{\Delta}}\right)^{1 / 2} \operatorname{erf}\left[\left(\tau_{\Delta}-1\right)\left(\frac{\delta_{\Delta}}{2 \Delta}\right)^{1 / 2}\right] .
\end{gathered}
$$

We see now that we recover the scaling law $\tau_{\Delta}^{2} \delta_{\Delta} \approx 2 \pi \Delta R_{\Delta}(0)^{2}$ [Eq. (170)] provided that $\delta_{\Delta}\left(\tau_{\Delta}-1\right)^{2} /(2 \Delta) \rightarrow \infty$ as $\Delta \rightarrow 0$. We have already had to impose $\delta_{\Delta} / \Delta \rightarrow \infty$ and $\left(\tau_{\Delta}-1\right) / \Delta \rightarrow \infty$, so it is likely that this requirement will be met.
We close our discussion by noting that if it were the case that $\delta_{\Delta}\left(\tau_{\Delta}-1\right)^{2} /(2 \Delta) \rightarrow 0$ as $\Delta \rightarrow 0$, then we would require $\tau_{\Delta} \rightarrow 1$ sufficiently rapidly to overcome the divergence of $\delta_{\Delta}\left(\tau_{\Delta}-1\right)^{2} /(2 \Delta)$ and correspondingly $\delta_{\Delta} \rightarrow 1 / 2$. In this case it would follow from Eq. (B.3) that $R_{\Delta}(0) \rightarrow 1$ and we would lose our non-constant periodic solution in the limit $\Delta \rightarrow 0$.

\section{Appendix C. Equilibrium with a power-law diffusivity and the potential $W(x)=\alpha|x|+\beta x^{2}$}

We sketch an analysis of the the determination of the equilibrium solution for a power-law diffusivity with the more general potential

$$
W(x)=\alpha|x|+\beta x^{2} .
$$

To reduce the prevalence of factors of $C$ in the analysis, where $C$ is the total mass present, defined by Eq. (2), in the remainder of this section only we shall work directly with an equilibrium mass density $\rho(x)$, rather than working with the probability density function $P(x)=\rho(x) / C$. From Eq. (182), we have

$$
\frac{D_{\eta}}{\eta}\left[\rho\left(\mu_{1}\right)^{\eta}-\rho(x)^{\eta}\right]=\int_{-\infty}^{\infty}\left[W(x-\xi)-W\left(\mu_{1}-\xi\right)\right] \rho(\xi) d \xi .
$$

Following the approach of Section 4.1, if we differentiate twice to obtain a delta function in the integrand, we obtain

$$
\frac{D_{\eta}}{\eta} \frac{d^{2}}{d x^{2}} \rho^{\eta}+2 \alpha \rho(x)+2 \beta C=0 .
$$

We now define

$$
R(x)=\rho(x)^{\eta}, \quad Q(x)=R^{\prime}(x),
$$

note that $R^{\prime \prime}(x)=(1 / 2)(d / d R)\left(Q^{2}\right)$, and find that

$$
\frac{D_{\eta}}{2 \eta} \frac{d}{d R}\left(Q^{2}\right)+2 \alpha R^{1 / \eta}+2 \beta C=0 .
$$

Integrating and taking $Q=0$ at $x=\mu_{1}$ we arrive at a first-order differential equation for $R(x)$ :

$\left(\frac{d R}{d x}\right)^{2}=\frac{4 \eta}{D_{\eta}}\left\{\beta C\left[R\left(\mu_{1}\right)-R(x)\right]+\frac{\alpha \eta\left[R\left(\mu_{1}\right)^{1+1 / \eta}-R(x)^{1+1 / \eta}\right]}{1+\eta}\right\}$.

Hence

$$
\begin{aligned}
& \left|x-\mu_{1}\right|=\left(\frac{D_{\eta}}{4 \eta}\right)^{1 / 2} \\
& \times \int_{R(x)}^{R\left(\mu_{1}\right)}\left\{\beta C\left[R\left(\mu_{1}\right)-z\right]+\frac{\alpha \eta\left[R\left(\mu_{1}\right)^{1+1 / \eta}-z^{1+1 / \eta}\right]}{1+\eta}\right\}^{-1 / 2} d z .
\end{aligned}
$$

The analysis just performed is only valid if the term in braces in the preceding equation is non-negative for $0 \leq z \leq R\left(\mu_{1}\right)$, which requires this term to be positive at $z=0$ and have a negative $z$-derivative for $0 \leq z<R\left(\mu_{1}\right)$, that is,

$$
R\left(\mu_{1}\right)>0, \quad \beta C+\frac{\alpha \eta R\left(\mu_{1}\right)^{1 / \eta}}{1+\eta}>0
$$


and

$$
\beta C+\alpha z^{1 / \eta}>0 \text { for } 0<z<R\left(\mu_{1}\right) .
$$

Subject to these conditions, we obtain a finite integral on the right-hand side of Eq. (C.4) as $R(x) \rightarrow 0$, leading to a density with finite support $\left(\mu_{1}-L, \mu_{1}+L\right)$, where

$$
\begin{aligned}
L & =\left[\frac{D_{\eta} R\left(\mu_{1}\right)}{4 \eta}\right]^{1 / 2} \\
& \times \int_{0}^{1}\left\{\beta C(1-Z)+\frac{\alpha \eta R\left(\mu_{1}\right)^{1 / \eta}\left(1-Z^{1+1 / \eta}\right)}{1+\eta}\right\}^{-1 / 2} d Z .
\end{aligned}
$$

From Eqs (C.4) and (C.7), we have

$$
\begin{aligned}
& \frac{\left|x-\mu_{1}\right|}{L} \\
& =\frac{\int_{R(x) / R\left(\mu_{1}\right)}^{1} \frac{d Z}{\sqrt{(1+\eta) \beta C(1-Z)+\alpha \eta R\left(\mu_{1}\right)^{1 / \eta}\left(1-Z^{1+1 / \eta}\right)}}}{\int_{0}^{1} \frac{d Z}{\sqrt{(1+\eta) \beta C(1-Z)+\alpha \eta R\left(\mu_{1}\right)^{1 / \eta}\left(1-Z^{1+1 / \eta}\right)}}} .
\end{aligned}
$$

Equations (C.7) and (C.8), together with

$$
\rho(x)=R(x)^{1 / \eta}, \quad \int_{\mu_{1}-L}^{\mu_{1}+L} \rho(x) d x=C,
$$

constitute a solution of the equilibrium problem, subject to the conditions (C.5) and (C.6), though for general values of $\eta$ the integrals have to be evaluated numerically.

If $\alpha \geq 0, \beta \geq 0$ and and at least one of $\alpha$ or $\beta$ is positive, the conditions (C.5) and (C.6) are met. The special case $\alpha=0$, $\beta>0$ has already been analysed in Section 7.3 and the results given there can be recovered easily from Eqs (C.7), (C.8) and (C.9). For the special case $\alpha>0, \beta=0$, we find that

$$
\frac{\left|x-\mu_{1}\right|}{L}=\left\{\int_{R(x) / R\left(\mu_{1}\right)}^{1} \frac{d Z}{\sqrt{1-Z^{1+1 / \eta}}}\right\} /\left\{\int_{0}^{1} \frac{d Z}{\sqrt{1-Z^{1+1 / \eta}}}\right\}
$$

which can be rewritten as

$$
\frac{\left|x-\mu_{1}\right|}{L}=\left\{\int_{\left[\rho(x) / \rho\left(\mu_{1}\right)\right]^{1+\eta}}^{1} \frac{\zeta^{\eta /(1+\eta)-1} d \zeta}{\sqrt{1-\zeta}}\right\} \mid\left\{\int_{0}^{1} \frac{\zeta^{\eta /(1+\eta)-1} d \zeta}{\sqrt{1-\zeta}}\right\} .
$$

The integrals are related to the classical beta function.

We turn now to the case in which $\alpha$ and $\beta$ are of opposite sign. When $\beta>0$ and $\alpha<0$, the condition (C.6) reduces to $|\alpha| R\left(\mu_{1}\right)^{1 / \eta}<\beta C$ and the second condition in (C.5) is then met. On the other hand, when $\beta<0$ and $\alpha>0$, the condition (C.6) necessarily fails in the vicinity of $z=0$.

We conclude our discussion with the special case in which $\eta=1$, that is, $\mathrm{D}(\rho)=D_{1} \rho$, in which case $R(x)=\rho(x)$. After some elementary but protracted algebra, we find that Eq. (C.8) can be reduced to

$$
\frac{\left|x-\mu_{1}\right|}{L}=\frac{\int_{0}^{\Phi(x)} \frac{d T}{\sqrt{1-\operatorname{sgn}(\alpha) T^{2}}}}{\int_{0}^{\Phi\left(\mu_{1}+L\right)} \frac{d T}{\sqrt{1-\operatorname{sgn}(\alpha) T^{2}}}}
$$

where

$$
\Phi(x)=\left\{\frac{|\alpha| \rho\left(\mu_{1}\right)\left[1-\rho(x) / \rho\left(\mu_{1}\right)\right]}{2\left[\alpha \rho\left(\mu_{1}\right)+\beta C\right]}\right\}^{1 / 2} .
$$

Hence for $\alpha>0$ and $\beta>0$,

$$
\begin{aligned}
\frac{\rho(x)}{\rho\left(\mu_{1}\right)}=1 & -\frac{2\left[\alpha \rho\left(\mu_{1}\right)+\beta C\right]}{\alpha \rho\left(\mu_{1}\right)} \\
& \times \sin ^{2}\left\{\frac{\left|x-\mu_{1}\right|}{L} \arcsin \left[\left(\frac{\alpha \rho\left(\mu_{1}\right)}{\left.2\left[\alpha \rho\left(\mu_{1}\right)\right)+\beta C\right]}\right)^{1 / 2}\right]\right\},
\end{aligned}
$$

while if $\alpha<0$ with $|\alpha| \rho\left(\mu_{1}\right)<\beta C$, we find that

$$
\begin{aligned}
\frac{\rho(x)}{\rho\left(\mu_{1}\right)}=1 & -\frac{2\left[\alpha \rho\left(\mu_{1}\right)+\beta C\right]}{|\alpha| \rho\left(\mu_{1}\right)} \\
& \times \sinh ^{2}\left\{\frac{\left|x-\mu_{1}\right|}{L} \operatorname{arcsinh}\left[\left(\frac{|\alpha| \rho\left(\mu_{1}\right)}{\left.2\left[\alpha \rho\left(\mu_{1}\right)\right)+\beta C\right]}\right)^{1 / 2}\right]\right\} .
\end{aligned}
$$

The solution is uniquely prescribed on selecting any one of the three constants $\rho\left(\mu_{1}\right), L$ or $C$, since we have the integral constraint (C.9) and we can show also that

$$
L=\left(\frac{2 D_{1}}{|\alpha|}\right)^{1 / 2} \times \begin{cases}\arcsin \left[\left(\frac{\alpha \rho\left(\mu_{1}\right)}{\left.2\left[\alpha \rho\left(\mu_{1}\right)\right)+\beta C\right]}\right)^{1 / 2}\right], & \alpha>0, \\ \operatorname{arcsinh}\left[\left(\frac{\alpha \mid \rho\left(\mu_{1}\right)}{\left.2\left[\alpha \rho\left(\mu_{1}\right)\right)+\beta C\right]}\right)^{1 / 2}\right], & \alpha<0 .\end{cases}
$$

\section{References}

[1] R. Eftimie, Hyperbolic and kinetic models for self-organized biological aggregations and movement: a brief review, Journal of Mathematical Biology 65 (2012) 35-75.

[2] D.J.T. Sumpter, The principles of collective animal behaviour, Philosophical Transactions of the Royal Society of London Series B 361 (2006) $5-22$.

[3] C.A. Yates, R. Erban, C. Escudero, I.D. Couzin, J. Buhl, I.G. Kevrekidis, P.K. Maini and D.J.T. Sumpter, Inherent noise can facilitate coherence in collective swarm motion, Proceedings of the National Academy of Sciences USA 106 (2009) 5464-5469.

[4] V. Guttal and I.D. Couzin, Social interactions, information use, and the evolution of collective migration, Proceedings of the National Academy of Sciences USA 107 16172-16177.

[5] K. Fellner and G. Raoul, Stability of stationary states of non-local interaction equations Mathematical Models and Methods in Applied Sciences 20 (2010) 2267-2291.

[6] K. Fellner and G. Raoul, Stability of stationary states of non-local equations with singular interaction potentials, Mathematical and Computer Modelling 53 (2011) 1436-1450.

[7] E.J. Hackett-Jones, K.A. Landman and K. Fellner, Aggregation patterns from non-local interactions: discrete stochastic and continuum modelling, Physical Review E 85 (2012) 041912.

[8] A.L Bertozzi, J.A . Carrillo and T. Laurent, Blow-up in multidimensional aggregation equations with mildly singular interaction kernels, Nonlinearity 22 (2009) 683-710.

[9] J.A. Carrillo, M. Di Francesco, A. Figalli, T. Laurent and D. Slepčev, Global-in-time weak measure solutions and finite-time aggregation for nonlocal interaction equations, Duke Mathematical Journal 156 (2011), 229-271.

[10] P. Biler and W.A. Woyczyński, Global and exploding solutions for nonlocal quadratic evolution problems, SIAM Journal on Applied Mathematics 59 (1998) 845-869

[11] M. Burger and M. Di Francesco, Large time behavior of nonlocal aggregation models with non-linear diffusion, Networks and Heterogeneous Media 3 (2008) 749-785. 
[12] D. Morale, V. Capasso and K. Oelschlager, An interacting particle system modelling aggregation behavior: from individuals to populations, Journal of Mathematical Biology 50, 49 (2005) 49-66.

[13] M. Bodnar and J. J. L. Velázquez, Derivation of macroscopic equations for individual cell-based models: a formal approach, Mathematical Methods in the Applied Sciences 28, 1757 (2005) 1757-1779.

[14] A.L. Bertozzi and J. Brandman, Finite-time blow-up of $L^{\infty}$-weak solutions of an aggregation equation, Communications in Mathematical Sciences 8 (2010) 45-65.

[15] A.L. Bertozzi and T. Laurent, Finite-time blow-up of solutions of an aggregation equation in $R^{n}$, Communications in Mathematical Physics 274 (2007). 717-735

[16] Y.-L. Chuang, Y. R. Huang, M. R. D’Orsogna and A. L. Bertozzi, Multivehicle flocking: scalability of cooperative control algorithms using pairwise potentials, IEEE International Conference on Robotics and Automation (2007) 2292-2299.

[17] C.M. Topaz, A.L. Bertozzi and M.A. Lewis, A nonlocal continuum mode for biological aggregation, Bulletin of Mathematical Biology 68, 1601 (2006)1601-1623

[18] A.J. Bernoff and C.M. Topaz, A primer of swarm equilibria, SIAM Journal on Applied Dynamical Systems 1 (2011) 212-250.

[19] D.D. Holm and V. Putkaradze, Aggregation of finite-size particles with variable mobility, Physical Review Letters 95 (2005) 226106.

[20] D.D. Holm and V. Putkaradze, Formation of clumps and patches in selfaggregation of finite-size particles, Physica D 220 (2006) 183-196.

21] J.A. Carrillo, R.J. McCann and C. Villani, Kinetic equilibration rates for granular media and related equations: entropy dissipation and mass transportation estimates. Revista Matemática Iberoamericana 19 (2003) 9711018

[22] F. Bolley, I. Gentil and A. Guillin, Uniform convergence to equilibrium for granular media. Archive for Rational Mechanics and Analysis 208 (2013) 429-445.

[23] N. J. Armstrong, K. J. , Painter and J. A. Sherratt, A continuum approach to modeling cell-cell adhesion. Journal of Theoretical Biology 243 (2006) 98-113.

[24] N.J. Armstrong, K.J. Painter and J.A. Sherratt, Adding adhesion to a chemical signaling model for somite formation. Bulletin of Mathematical Biology 71 (2009) 124.

[25] G. Civelekoglu and L. Edelstein-Keshet, Modelling the dynamics of Factin in the cell, Bulletin of Mathematical Biology 56 (1994) 587-616.

[26] K. Kang, B. Perthame, A. Stevens and J.J.L. Velázquez, An integrodifferential equation model for alignment and orientational aggregation, Journal of Differential Equations 246 (2009) 1387-1421.

[27] I. Primi, A. Stevens and J.J.L. Velázquez, Mass-selection in alignmen models with non-deterministic effects, Communications in Partial Differential Equations 34 (2009) 419-456.

[28] A. Mogilner and L. Edelstein-Keshet, A non-local model for a swarm, Journal of Mathematical Biology 38 (1999) 534-570.

[29] D. Balagué, J.A. Carrillo, T. Laurent and G. Raoul, Nonlocal interaction by repulsive-attractive potentials: radial ins/stability, arViv: $1109.5258 \mathrm{v} 1$ 24 September 2011.

[30] J. Crank, The Mathematics of Diffusion, 2nd edition, Oxford University Press (1979).

[31] B.D. Hughes, Random Walks and Random Environments, Vol. 1, Oxford University Press (1995)

[32] R. Metzler and J. Klafter, The random walk's guide to anomalous diffusion: a fractional dynamics approach, Physics Reports 339 (2000) 1-77.

[33] C.J. Penington, B.D. Hughes and K.A. Landman, Building macroscale models from microscale probabilistic models: a general probabilistic approach for nonlinear diffusion and multi-species phenomena, Physical Review E 84 (2011) 041120.

[34] M.J. Simpson, K.A. Landman and B.D. Hughes, Multi-species simple exclusion processes Physica A 388, (2009) 399-406.

[35] M. J. Simpson, K. A. Landman, B. D. Hughes and A. E. Fernando, A model for mesoscale patterns in motile populations, Physica A 389 (2010) 1412-1424

[36] A. E. Fernando, K. A. Landman and M. J. Simpson, Nonlinear diffusion and exclusion processes with contact interactions, Physical Review E 81 (2010) 011903

[37] K. A. Landman and A. E. Fernando, Myopic random walkers and exclusion processes: single and multispecies, Physica A 390 (2011) 3742
3753.

[38] T. M. Liggett, Stochastic Interacting Systems: Contact, Voter and Exclusion Processes, Springer-Verlag, Berlin (1999).

[39] M.J. Simpson and R.E. Baker, Corrected mean-field models for spatially dependent advection-diffusion-reaction phenomena, Physical Review E 83 (2011) 051922.

[40] H.G. Othmer and A Stevens, Aggregation, blowup and collapse: the ABC's of taxis in reinforced random walks, SIAM Journal on Applied Mathematics 57(1997) 1044-1081

[41] K.J. Painter and J.A. Sherratt, Modelling the movement of interacting cell populations, Journal of Theoretical Biology 225 (2003) 327-339.

[42] T. Hillen and K. J. Painter, A users guide to PDE models for chemotaxis, Journal of Mathematical Biology 58 (2009) 183-217.

[43] R. Klages, G. Radons and I.M. Sokolov (editors), Anomalous Transport, Weinheim, Wiley-VCH (2008).

[44] P. Biler and G. Karch, Blowup of solutions to generalzed Keller-Segel model, Journal of Evolution Equations 10 (2010) 247-267.

[45] R. Gorenflo and F. Mainardi, Continuous-time random walk, MittagLeffler waiting time and fractional diffusion: mathematical aspects, in [43], pp. 93-127.

[46] B.D. Hughes, Anomalous diffusion, stable processes, and generalized functions, Physical Review E 65 (2002) 035105(R).

[47] F.W.J. Olver, D.W. Lozier, R.F. Boisvert and C.W. Clark (editors), NIST Handbook of Mathematical Functions, Cambridge University Press (2010).

[48] E. Geigant and M. Stoll Stability of peak solutions of a non-linear transport equation on the circle. Electronic Journal of Differential Equations 2012 (2012), 157 (41pp.).

[49] M.J. Lighthill, Introduction to Fourier Analysis and Generalised Functions, Cambridge University Press (1958).

[50] F.W.J. Olver, Asymptotics and Special Functions, New York, Academic Press (1974).

[51] B.I. Henry, T.A.M. Langlands and S.L. Wearne, Anomalous diffusion with linear reaction dynamics: from continuous time random walks to fractional reaction-diffusion equations, Physical Review E 74 (2006) 031116. 


\section{University Library}

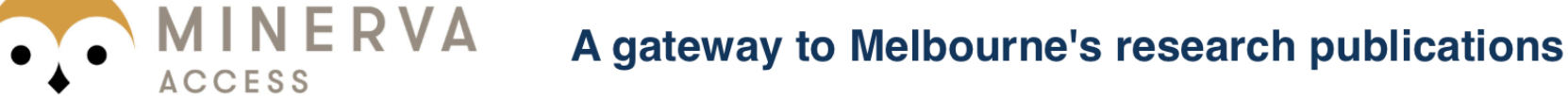

Minerva Access is the Institutional Repository of The University of Melbourne

Author/s:

Hughes, BD;Fellner, K

Title:

Continuum models of cohesive stochastic swarms: The effect of motility on aggregation patterns

Date:

2013-10-01

Citation:

Hughes, B. D. \& Fellner, K. (2013). Continuum models of cohesive stochastic swarms: The effect of motility on aggregation patterns. PHYSICA D-NONLINEAR PHENOMENA, 260, pp.26-48. https://doi.org/10.1016/j.physd.2013.05.001.

Persistent Link:

http://hdl.handle.net/11343/58831 